\title{
Stability of Rarefaction Waves to the 1D Compressible Navier-Stokes Equations with Density-dependent Viscosity
}

\author{
Quansen Jiu, ${ }^{1,3 *}$ Yi Wang ${ }^{2,3 \dagger}$ and Zhouping $\mathbf{X i n}^{3 \ddagger}$ \\ ${ }^{1}$ School of Mathematical Sciences, Capital Normal University, Beijing 100048, P.R. China \\ ${ }^{2}$ Institute of Applied Mathematics, AMSS, CAS, Beijing 100190, P. R. China \\ ${ }^{3}$ The Institute of Mathematical Sciences, CUHK, Shatin N.T., HongKong
}

\begin{abstract}
In this paper, we study the asymptotic stability of rarefaction waves for the compressible isentropic Navier-Stokes equations with density-dependent viscosity. First, a weak solution around a rarefaction wave to the Cauchy problem is constructed by approximating the system and regularizing the initial values which may contain vacuum state. Then some global in time estimates on the weak solution are obtained. Based on these uniform estimates, the vacuum states are shown to vanish in finite time and the weak solution we constructed becomes a unique strong one. Consequently, the stability of the rarefaction wave is proved in a weak sense. The theory holds for large-amplitudes rarefaction waves and arbitrary initial perturbations.
\end{abstract}

Key words: density-dependent Navier-Stokes equations, rarefaction wave, weak solution, stability

AMS SC2000: 35L60, 35L65

\section{Introduction}

In this paper, we consider the following compressible and isentropic Navier-Stokes equations with density-dependent viscosity

$$
\left\{\begin{array}{l}
\rho_{t}+(\rho u)_{x}=0, \\
(\rho u)_{t}+\left(\rho u^{2}+p(\rho)\right)_{x}=\left(\mu(\rho) u_{x}\right)_{x},
\end{array} \quad x \in \mathbf{R}, t>0\right.
$$

${ }^{*}$ The research is partially supported by National Natural Sciences Foundation of China (No. 10871133) and Project of Beijing Education Committee. E-mail: qsjiumath@gmail.com

${ }^{\dagger}$ The research is partially supported by National Natural Sciences Foundation of China (No. 10801128). E-mail: wangyi@amss.ac.cn.

¥The research is partially supported by Zheng Ge Ru Funds, Hong Kong RGC Earmarked Research Grant CUHK4042/08P and CUHK4040/06P, and a Focus Area Grant at The Chinese University of Hong Kong. Email: zpxin@ims.cuhk.edu.hk 
where $\rho(t, x) \geq 0, u(t, x)$ represent the density and the velocity of the gas, respectively. Assume that the pressure and viscosity function is given by

$$
p(\rho)=A \rho^{\gamma}, \quad \mu(\rho)=B \rho^{\alpha},
$$

where $\gamma>1$ denotes the adiabatic exponent, $\alpha>0$ and $A, B>0$ are the gas constants. Without loss of generality, we assume that $A=B=1$.

We consider the Cauchy problem (1.1) with the initial values

$$
(\rho, \rho u)(0, x)=\left(\rho_{0}, m_{0}\right)(x) \rightarrow\left(\rho_{ \pm}, m_{ \pm}\right) \text {as } x \rightarrow \pm \infty,
$$

where $\rho_{ \pm}, m_{ \pm}$are given constants. Here we assume that there is no vacuum at the far field, i.e., $\rho_{ \pm}>0$, thus we can define the far field velocity by $u_{ \pm}=\frac{m_{ \pm}}{\rho_{ \pm}}$.

The large time asymptotic behavior of solutions to (1.1) is expected to be closely related to that of the corresponding Euler system

$$
\left\{\begin{array}{l}
\rho_{t}+(\rho u)_{x}=0 \\
(\rho u)_{t}+\left(\rho u^{2}+p(\rho)\right)_{x}=0
\end{array}\right.
$$

The Euler system (1.4) is a strictly hyperbolic one for $\rho>0$ whose characteristic fields are both genuinely nonlinear, that is, in the equivalent system

$$
\left(\begin{array}{l}
\rho \\
u
\end{array}\right)_{t}+\left(\begin{array}{cc}
u & \rho \\
p^{\prime}(\rho) / \rho & u
\end{array}\right)\left(\begin{array}{l}
\rho \\
u
\end{array}\right)_{x}=0
$$

the Jacobi matrix

$$
\left(\begin{array}{cc}
u & \rho \\
p^{\prime}(\rho) / \rho & u
\end{array}\right)
$$

has two distinct eigenvalues

$$
\lambda_{1}(\rho, u)=u-\sqrt{p^{\prime}(\rho)}, \quad \lambda_{2}(\rho, u)=u+\sqrt{p^{\prime}(\rho)}
$$

with corresponding right eigenvectors

$$
r_{i}(\rho, u)=\left(1,(-1)^{i} \frac{\sqrt{p^{\prime}(\rho)}}{\rho}\right)^{t}, \quad i=1,2,
$$

such that

$$
r_{i}(\rho, u) \cdot \nabla_{\rho, u} \lambda_{i}(\rho, u)=(-1)^{i} \frac{\rho p^{\prime \prime}(\rho)+2 p^{\prime}(\rho)}{2 \rho \sqrt{p^{\prime}(\rho)}} \neq 0, \quad i=1,2 .
$$

Define the $i$-Riemann invariant $(i=1,2)$ by

$$
\Sigma_{i}(\rho, u)=u+(-1)^{i} \int^{\rho} \frac{\sqrt{p^{\prime}(s)}}{s} d s
$$

such that

$$
\nabla_{(\rho, u)} \Sigma_{i}(\rho, u) \cdot r_{i}(\rho, u) \equiv 0, \quad \forall \rho>0, u
$$


There are two families of rarefaction waves to the Euler system (1.4). Here we only consider 2 -rarefaction wave, which is characterized by the fact that 2 -Riemann invariant $\Sigma_{2}(\rho, u)$ is constant in $(x, t)$ and 2 -characteristic speed, $\lambda_{2}(\rho, u)$ is increasing in $x$. Suppose that the end states $\left(\rho_{ \pm}, u_{ \pm}\right)$of the initial values of (1.4) satisfy

$$
\Sigma_{2}\left(\rho_{-}, u_{-}\right)=\Sigma_{2}\left(\rho_{+}, u_{+}\right), \quad \lambda_{2}\left(\rho_{+}, u_{+}\right)>\lambda_{2}\left(\rho_{-}, u_{-}\right),
$$

Then the state $\left(\rho_{-}, u_{-}\right)$can be connected to the state $\left(\rho_{+}, u_{+}\right)$by a 2 -rarefaction wave. The 2 -rarefaction waves for (1.4) connecting $\left(\rho_{-}, u_{-}\right)$to $\left(\rho_{+}, u_{+}\right)$converge to each other timeasymptotically in supreme norm. For definiteness, we choose a particular 2-rarefaction wave $(\bar{\rho}, \bar{u})(t, x)$ of (1.4) defined by (2.3) in the next section.

In the present paper, we are interested in the large time-asymptotic stability of the above rarefaction wave to the density-dependent Navier-Stokes equation (1.1) with large initial values which may contain vacuum states.

There have been large literature on the global existence and the large time behavior of solutions to the system (1.1) and even in the higher dimensional case when the viscosity $\mu(\rho)$ is constant. We refer to [7], [15], [17], [19], 20], 23], 24] and the references therein. However, the possible appearance of the vacuum is one of the major difficulties to the global existence of the solutions to the compressible Navier-Stokes equations with constant viscosity. Hoff and Smoller 13] proved that weak solutions of the compressible Navier-Stokes equations in 1D case do not contain vacuum states in finite time if there is no vacuum initially. On the other hand, Xin [30] proved that there is no global smooth solution to the Cauchy problem to compressible Navier-Stokes equations with a nontrivial compactly supported initial density, which means that the solution will blow up in finite time in the presence of the vacuum states. Moreover, Hoff [11] proved the results of the failure of the continuous dependence of the weak solutions containing vacuum states on the initial values.

Thus, when the solution may contain vacuum states, it is natural to consider the compressible Navier-Stokes equations with density-dependent viscosity, as was derived from Chapman-Enskog expansions from the Boltzmann equation where the viscosity depends on the temperature and thus on the density for isentropic flows. Moreover, a one-dimensional viscous Saint-Venant system for the shallow water, derived rigorously from the incompressible Navier-Stokes equation with a moving free surface by Gerbeau-Perthame recently in [8], is expressed exactly as in (1.1)-(1.2) with $\alpha=1$ and $\gamma=2$.

There are many literatures on mathematical studies on (1.1)-(1.2). If the initial density is assumed to be connected to vacuum with discontinuities, Liu, Xin and Yang first obtained in [21] the local existence of weak solutions. The global existence of weak solutions was obtained later by [14, [15], 27], 31] respectively. If the initial density connects to vacuum continuously, then new difficulty is encountered since no positive lower bound for the density is available. This case is studied by [6], 29], 31] and [32 respectively. However, most of these results concern with free boundary problems. Recently, initial-boundary-value problems for one-dimensional equations (1.1)-(1.2) with $\mu(\rho)=\rho^{\alpha}(\alpha>1 / 2)$ was studied by Li, Li and Xin in [18, and the phenomena of vacuum vanishing and blow-up of solutions were found there. The global existence of weak solutions for the initial-boundary-value problems for spherically symmetric compressible NavierStokes equations with density-dependent viscosity was proved by Guo, Jiu and Xin in [9]. More recently, there are some results on Cauchy problem (1.1)-(1.3). The existence and uniqueness of global strong solutions to the compressible Navier-Stokes equations (1.1)-(1.3) were obtained by 
Mellet and Vasseur 25] where no vacuum is permitted in the initial density. However, the a priori estimates obtained in [25] depends on the time interval thus does not yield the time-asymptotic behavior of the solutions. The first result about the time-asymptotic behavior of the solutions to the Cauchy problem (1.1)-(1.3) is obtained by Jiu-Xin [16], where the global existence, large time-asymptotic behavior, the vanishing of the vacuum and the blow-up phenomena of the weak solutions were considered in the case that $\rho_{+}=\rho_{-}$and $u_{+}=u_{-}=0$. It is well-known that the large time-asymptotic behavior of solutions to the system (1.1)-(1.3) with different far field states of the initial values is closely related to the corresponding Euler system (1.4). Rarefaction wave is one of the fundamental wave patterns to the Euler system (1.4). A natural question is how about the stability of rarefaction waves to the compressible Navier-Stokes equations (1.1)-(1.3) in the framework of weak solutions which may contain vacuum states.

In the case $\rho_{ \pm}>0$, that is, the rarefaction wave does not contain vacuum, we study in this paper the global existence, large time-asymptotic behavior, vanishing of the vacuum and the blow-up phenomena of weak solutions to the Cauchy problem (1.1)-(1.3). First we will construct a class of approximate solutions satisfying some uniform estimates and furthermore prove the global existence of weak solutions for Cauchy problem of (1.1)-(1.3). Moreover, the time-asymptotic behaviors of weak solutions are investigated. More precisely, it is proved that the density $\rho$ tends to a rarefaction wave as $t \rightarrow \infty$. As a consequence, there exists a time $T_{0}>0$ such that when $t>T_{0}$, the vacuum states vanish and the global weak solution becomes a unique strong one. Moreover, the stability of the rarefaction wave is obtained in some weak sense.

Notations. Throughout this paper, positive generic constants are denoted by $c$ and $C$, which are independent of $\varepsilon, t$ and $T$, without confusion, and $C(\cdot)$ stands for some generic constant(s) depending only on the quantity listed in the parenthesis. For function spaces, $L^{p}(\Omega), 1 \leq p \leq \infty$, denote the usual Lebesgue spaces on $\Omega \subset \mathbb{R}:=(-\infty, \infty)$. $W^{k, p}(\Omega)$ denotes the $k^{\text {th }}$ order Sobolev space, $H^{k}(\Omega):=W^{k, 2}(\Omega),\|\cdot\|:=\|\cdot\|_{L^{2}(\Omega)}$, and $\|\cdot\|_{k}:=\|\cdot\|_{H^{k}(\Omega)}$ for simplicity. The domain $\Omega$ will be often abbreviated without confusion.

\section{Preliminaries and Main Results}

\subsection{Rarefaction waves}

Consider the solution to the following Cauchy problem for Burgers equation

$$
\left\{\begin{array}{l}
w_{t}+w w_{x}=0, \quad t>0, x \in \mathbb{R} \\
w_{0}(x):=w(0, x)=\frac{w_{+}+w_{-}}{2}+\frac{w_{+}-w_{-}}{2} K_{q} \int_{0}^{\eta x}\left(1+y^{2}\right)^{-q} d y
\end{array}\right.
$$

Here $q \geq 2$ is some fixed constant, and $K_{q}$ is a constant such that $K_{q} \int_{0}^{\infty}\left(1+y^{2}\right)^{-q} d y=1$, and $\eta$ is a small positive constant to be determined later. It is easy to see that the solution to the above Burgers equation is given by

$$
w(t, x)=w_{0}\left(x_{0}(t, x)\right), \quad x=x_{0}(t, x)+w_{0}\left(x_{0}(t, x)\right) t .
$$

Then the following properties hold (see [24]). 
Lemma 2.1 Let $0 \leq w_{-}<w_{+}$, Burgers equation (2.1) has a unique smooth solution $w(t, x)$ satisfying

i) $w_{-}<w(t, x)<w_{+}, w_{x}(t, x)>0$,

ii) For any $p(1 \leq p \leq \infty)$, there exists a constant $C_{p q}$ such that

$$
\begin{aligned}
& \left\|w_{x}(t)\right\|_{L^{p}} \leq C_{p q} \min \left\{\delta_{r} \eta^{1-\frac{1}{p}}, \delta_{r}^{\frac{1}{p}} t^{-1+\frac{1}{p}}\right\} \\
& \left\|w_{x x}(t)\right\|_{L^{p}} \leq C_{p q} \min \left\{\delta_{r} \eta^{2-\frac{1}{p}}, \eta^{\left(1-\frac{1}{2 q}\right)\left(1-\frac{1}{p}\right)} \delta_{r}^{-\frac{(p-1)}{2 p q}} t^{-1-\frac{(p-1)}{2 p q}}\right\},
\end{aligned}
$$

where $\delta_{r}=w_{+}-w_{-}$,

iii) $\sup _{x \in \mathbf{R}}\left|w(t, x)-w^{r}\left(\frac{x}{t}\right)\right| \rightarrow 0$, as $t \rightarrow \infty$.

Define a 2 -rarefaction wave $(\bar{\rho}, \bar{u})(t, x)$ by

$$
\begin{aligned}
& \lambda_{2}\left(\rho_{ \pm}, u_{ \pm}\right)=w_{ \pm}, \quad w_{-}<w_{+}, \\
& \lambda_{2}(\bar{\rho}(t, x), \bar{u}(t, x))=w(1+t, x), \\
& \Sigma_{2}(\bar{\rho}(t, x), \bar{u}(t, x))=\Sigma_{2}\left(\rho_{ \pm}, u_{ \pm}\right),
\end{aligned}
$$

Thus $(\bar{\rho}, \bar{u})(t, x)$ satisfies the system

$$
\left\{\begin{array}{l}
\bar{\rho}_{t}+(\bar{\rho} \bar{u})_{x}=0 \\
(\bar{\rho} \bar{u})_{t}+\left(\bar{\rho} \bar{u}^{2}+p(\bar{\rho})\right)_{x}=0
\end{array}\right.
$$

Lemma 2.2 The 2 -rarefaction wave $(\bar{\rho}, \bar{u})(t, x)$ satisfies

i) $\bar{\rho}_{x}>0, \quad \bar{u}_{x}>0$

ii) For any $p(1 \leq p \leq \infty)$, there exists a constant $C_{p q}$ such that

$$
\begin{aligned}
& \left\|\left(\bar{\rho}_{x}, \bar{u}_{x}\right)(t, \cdot)\right\|_{L^{p}(\mathbf{R})} \leq C_{p q} \min \left\{\delta \eta^{1-\frac{1}{p}}, \delta^{\frac{1}{p}}(1+t)^{-1+\frac{1}{p}}\right\}, \\
& \left\|\left(\bar{\rho}_{x x}, \bar{u}_{x x}\right)(t, \cdot)\right\|_{L^{p}(\mathbf{R})} \leq C_{p q} \min \left\{\delta \eta^{2-\frac{1}{p}}, \eta^{\left(1-\frac{1}{2 q}\right)\left(1-\frac{1}{p}\right)} \delta^{-\frac{p-1}{2 p q}}(1+t)^{-1-\frac{p-1}{2 p q}}+\delta^{\frac{1}{p}}(1+t)^{-2+\frac{1}{p}}\right\},
\end{aligned}
$$

where $\delta=\left|\rho_{+}-\rho_{-}\right|+\left|u_{+}-u_{-}\right|$is the strength of the rarefaction wave;

iii) $\lim _{t \rightarrow \infty} \sup _{\xi \in \mathbf{R}}\left|(\bar{\rho}, \bar{u})(t, x)-\left(\rho^{r}, u^{r}\right)\left(\frac{x}{1+t}\right)\right|=0$.

Remark: For any $1<p \leq+\infty$,

$$
\int_{0}^{T}\left\|\left(\bar{\rho}_{x x}, \bar{u}_{x x}\right)(t, \cdot)\right\|_{L^{p}(\mathbf{R})} d t \leq C,
$$

where $C$ is independent of $T$. Note that in the case $p=1$, the constant $C$ in the above estimates is not uniform in $T$.

Moreover, the following estimate holds:

$$
\int_{0}^{T}\left\|\left(\bar{\rho}_{x x}, \bar{u}_{x x}\right)(t, \cdot)\right\|_{L^{\infty}(\mathbf{R})} d t \leq C \eta^{\frac{2}{4 q+1}} \int_{0}^{T}(1+t)^{-1-\frac{1}{4 q+1}} d t \leq C \eta^{\frac{2}{4 q+1}},
$$




\subsection{Main Results}

Set

$$
\begin{aligned}
\Psi(\rho, \bar{\rho}) & =\int_{\bar{\rho}}^{\rho} \frac{p(s)-p(\bar{\rho})}{s^{2}} d s \\
& =\frac{1}{(\gamma-1) \rho}\left[\rho^{\gamma}-\bar{\rho}^{\gamma}-\gamma \bar{\rho}^{\gamma-1}(\rho-\bar{\rho})\right]
\end{aligned}
$$

The initial data are assumed to satisfy:

$$
\left\{\begin{array}{c}
\rho_{0} \geq 0 ; \quad m_{0}=0 \quad \text { a.e.on }\left\{x \in \mathbf{R} \mid \rho_{0}(x)=0\right\} \\
\left(\rho_{0}^{\alpha-\frac{1}{2}}\right)_{x} \in L^{2}(\mathbf{R}), \quad \rho_{0} \Psi\left(\rho_{0}, \bar{\rho}_{0}\right) \in L^{1}(\mathbf{R}) \\
\rho_{0}\left(\frac{m_{0}}{\rho_{0}}-\bar{u}_{0}\right)^{2} \in L^{1}(\mathbf{R}), \quad \rho_{0}\left(\frac{m_{0}}{\rho_{0}}-\bar{u}_{0}\right)^{3} \in L^{1}(\mathbf{R})
\end{array}\right.
$$

where $\alpha>\frac{1}{2}$ and $\left(\bar{\rho}_{0}, \bar{u}_{0}\right):=(\bar{\rho}, \bar{u})(0, x)$ is the initial values of the 2 -rarefaction wave $(\bar{\rho}, \bar{u})(t, x)$ constructed in section 2.1. Note that (2.6) implies that $\rho_{0} \in C(\mathbf{R})$ which is the space of continuous functions.

Before stating the main results, we give the definition of weak solutions to (1.1)-(1.3) associated with 2 -rarefaction wave $(\bar{\rho}, \bar{u})(t, x)$ in (2.4) .

Definition 2.1. A pair $(\rho, u)$ is said to be a weak solution to (1.1)-(1.3) towards the rarefaction wave $(\bar{\rho}, \bar{u})(t, x)$ in (2.4), provided that

(1) $\rho \geq 0$ a.e., and

$$
\begin{aligned}
& \left.\rho \in L^{\infty}\left(0, T ; L^{\infty}(\mathbf{R})\right)\right) \cap C\left([0, \infty) ; W^{1, \infty}(\mathbf{R})^{*}\right), \\
& \left(\rho^{\alpha-\frac{1}{2}}\right)_{x} \in L^{\infty}\left(0, T ; L^{2}(\mathbf{R})\right), \sqrt{\rho}(u-\bar{u}) \in L^{\infty}\left(0, T ; L^{2}(\mathbf{R})\right),
\end{aligned}
$$

where $W^{1, \infty}(\mathbf{R})^{*}$ is the dual space of $W^{1, \infty}(\mathbf{R})$;

(2) For any $t_{2} \geq t_{1} \geq 0$ and any $\zeta \in C_{0}^{1}\left(\mathbf{R} \times\left[t_{1}, t_{2}\right]\right)$, the mass equation (1.1) holds in the following sense:

$$
\left.\int_{\mathbf{R}}(\rho-\bar{\rho}) \zeta d x\right|_{t_{1}} ^{t_{2}}=\int_{t_{1}}^{t_{2}} \int_{\mathbf{R}}\left[(\rho-\bar{\rho}) \zeta_{t}+(\rho u-\bar{\rho} \bar{u}) \cdot \zeta_{x}\right] d x d t
$$

(3) For any $\psi \in C_{0}^{\infty}(\mathbf{R} \times[0, T))$, it holds that

$$
\begin{aligned}
& \int_{\mathbf{R}}\left(m_{0}-\bar{\rho}_{0} \bar{u}_{0}\right) \psi(0, \cdot) d x+\int_{0}^{T} \int_{\mathbf{R}}\{\sqrt{\rho}[\sqrt{\rho}(u-\bar{u})]+(\rho-\bar{\rho}) \bar{u}\} \psi_{t} \\
& +\left\{[\sqrt{\rho}(u-\bar{u})]^{2}-2 \sqrt{\rho} \sqrt{\rho}(u-\bar{u}) \bar{u}+(\rho-\bar{\rho}) \bar{u}^{2}+\left(\rho^{\gamma}-\bar{\rho}^{\gamma}\right)\right\} \psi_{x} d x d t \\
& +<\rho^{\alpha}(u-\bar{u})_{x}, \psi_{x}>+\int_{0}^{T} \int_{\mathbf{R}} \rho^{\alpha} \bar{u}_{x} \psi_{x} d x d t=0,
\end{aligned}
$$

where the diffusion term makes sense when written as

$$
\begin{aligned}
& <\rho^{\alpha}(u-\bar{u})_{x}, \psi>=-\int_{0}^{T} \int_{R} \rho^{\alpha-\frac{1}{2}} \sqrt{\rho}(u-\bar{u}) \psi_{x} d x d t \\
& -\frac{2 \alpha}{2 \alpha-1} \int_{0}^{T} \int_{R}\left(\rho^{\alpha-\frac{1}{2}}\right)_{x} \sqrt{\rho}(u-\bar{u}) \psi d x d t .
\end{aligned}
$$


Our main results read as

Theorem 2.1 (Existence of a weak solution) Let $\alpha$ and $\gamma$ satisfy that

$$
\frac{1}{2}<\alpha \leq \frac{\gamma+1}{2}
$$

Suppose that (2.6) holds. Then the Cauchy problem (1.1)-(1.3) admits a global weak solution $(\rho(x, t), u(x, t))$ satisfying

$$
\begin{gathered}
\rho \in C(\mathbf{R} \times(0, T)), \\
\rho \geq 0, \quad \max _{(x, t) \in \mathbf{R} \times[0, T]} \rho \leq C, \\
\sup _{t \in[0, T]} \int_{\mathbf{R}}\left(|\sqrt{\rho}(u-\bar{u})|^{2}+\left(\rho^{\alpha-\frac{1}{2}}\right)_{x}^{2}+\frac{1}{\gamma-1}\left[\rho^{\gamma}-\bar{\rho}^{\gamma}-\gamma \bar{\rho}^{\gamma-1}(\rho-\bar{\rho})\right] d x\right. \\
+\int_{0}^{T} \int_{\mathbf{R}}\left(\left[\left(\rho^{\frac{\gamma+\alpha-1}{2}}-\bar{\rho}^{\frac{\gamma+\alpha-1}{2}}\right)_{x}\right]^{2}+\Lambda(x, t)^{2}\right) d x d t \leq C,
\end{gathered}
$$

where $C$ is an absolute constant depending on the initial data and $\Lambda(x, t) \in L^{2}(\mathbf{R} \times(0, T))$ satisfying

$$
\begin{aligned}
& \int_{0}^{T} \int_{\mathbf{R}} \Lambda \varphi d x d x t=-\int_{0}^{T} \int_{\mathbf{R}} \rho^{\alpha-\frac{1}{2}} \sqrt{\rho}(u-\bar{u}) \varphi_{x} d x d t \\
& -\frac{2 \alpha}{2 \alpha-1} \int_{0}^{T} \int_{\mathbf{R}}\left(\rho^{\alpha-\frac{1}{2}}\right)_{x} \sqrt{\rho}(u-\bar{u}) \varphi d x d t .
\end{aligned}
$$

Remark 1: Obviously, the case of shallow water equation, i.e. $\alpha=1, \gamma=2$, is included in our theorem.

Theorem 2.2 Suppose that $(\rho(x, t), u(x, t))$ is a weak solution of the Cauchy problem (1.1)(1.3) satisfying (2.12), (2.13) and (2.14). Then we have

$$
\lim _{t \rightarrow \infty} \sup _{x \in \mathbf{R}}|\rho-\bar{\rho}|=0 .
$$

Based on Theorem 2.2, it is easy to deduce that under the assumption that the approximate rarefaction wave satisfies $\inf _{x, t} \bar{\rho}(t, x)>0$, there exists a time $T_{0}>0$ after which the density has a positive lower bound and the vacuum states vanish. Moreover, it will be shown that after the time $t=T_{0}$, the weak solution becomes a unique strong one. Precisely, we have

Theorem 2.3 Suppose that the assumptions of Theorem 2.1 hold. Let $(\rho(x, t), u(x, t))$ be a weak solution of the Cauchy problem (1.1)-(1.3) satisfying (2.12),(2.13) and (2.14). Then for any $0<\rho_{1}<\inf _{t, x} \bar{\rho}(t, x)$, there exists a time $T_{0}$ such that

$$
0<\rho_{1} \leq \rho(x, t) \leq C, \quad(x, t) \in \mathbf{R} \times\left[T_{0}, \infty\right),
$$

where $C$ is a constant same as in (2.13). Moreover, for $t \geq T_{0}$, the weak solution becomes a unique strong solution to (1.1)-(1.3), satisfying

$$
\begin{aligned}
& \rho-\bar{\rho} \in L^{\infty}\left(T_{0}, t ; H^{1}(\mathbf{R})\right), \quad \rho_{t} \in L^{\infty}\left(T_{0}, t ; L^{2}(\mathbf{R})\right), \\
& u-\bar{u} \in L^{2}\left(T_{0}, t ; H^{2}(\mathbf{R})\right), \quad u_{t} \in L^{2}\left(T_{0}, t ; L^{2}(\mathbf{R})\right)
\end{aligned}
$$


and

$$
\sup _{x \in \mathbf{R}}|\rho-\bar{\rho}|+\|\rho-\bar{\rho}\|_{L^{p}(\mathbf{R})}+\|u-\bar{u}\|_{L^{2}(\mathbf{R})} \rightarrow 0
$$

as $t \rightarrow \infty$, where $2<p \leq \infty$.

Remark 2: It is interesting to note that there is no requirement on the sizes of the strength of the rarefaction wave and the perturbations. The class of initial perturbations given by (2.6) is quite large compared with those for the constant viscosity case, [20], 223], 24].

In addition, similar to [18, we can obtain some results on the blow-up phenomena of the solution when the vacuum states vanish, which will be presented in Section 5 .

\section{Existence of a weak solution}

We first study the following approximate system:

$$
\left\{\begin{array}{l}
\rho_{t}+(\rho u)_{x}=0, \\
(\rho u)_{t}+\left(\rho u^{2}+p(\rho)\right)_{x}=\left(\mu_{\varepsilon}(\rho) u_{x}\right)_{x},
\end{array} \quad x \in \mathbf{R}, \quad t>0,\right.
$$

where $\mu_{\varepsilon}(\rho)=\rho^{\alpha}+\varepsilon \rho^{\theta}, \varepsilon>0, \theta \in\left(0, \frac{1}{2}\right)$.

The initial values can be regularized in the following way. Due to (2.6), we have

$$
\lim _{x \rightarrow \pm \infty} \rho_{0}(x)=\rho_{ \pm}
$$

For any suitably small constant $\lambda>0$, there exists $M>0$ such that if $|x| \geq M$, then

$$
\rho_{0}(x) \geq \lambda
$$

For definiteness, we can choose and fix $\lambda=\frac{\rho_{-}}{2}$.

Define

$$
\rho_{0}^{1}(x):= \begin{cases}\rho_{0}(x)+\varepsilon^{\frac{1}{2 \alpha-2 \theta}}, & \text { if }|x| \leq M \\ \rho_{0}(x)+\varepsilon^{\frac{1}{2 \alpha-2 \theta}}[(M+1) \mp x], & \text { if } \quad M \leq \pm x \leq M+1 \\ \rho_{0}(x), & \text { if }|x| \geq M+1\end{cases}
$$

Then, $\rho_{0}^{1}(x)$ is a continuous function in $\mathbf{R}$ and $\rho_{0}^{1}(x) \geq \min \left\{\frac{\rho_{-}}{2}, \varepsilon^{\frac{1}{2 \alpha-2 \theta}}\right\}=\varepsilon^{\frac{1}{2 \alpha-2 \theta}}$ for suitably small $\varepsilon$. Also,

$$
0 \leq \rho_{0}^{1}(x)-\rho_{0}(x)=\left.\left(\rho_{0}^{1}(x)-\rho_{0}(x)\right) \mathbf{1}\right|_{|x| \leq M+1} \leq 2(M+1) \varepsilon^{\frac{1}{2 \alpha-2 \theta}} .
$$

Set

$$
\rho_{0 \varepsilon}(x)=\left(\rho_{0}^{1}-\bar{\rho}_{0}\right) * j_{\varepsilon}(x)+\bar{\rho}_{0}(x) .
$$

Hence, $\rho_{0 \varepsilon} \in C^{\infty}(\mathbf{R})$ and $\lim _{x \rightarrow \pm \infty} \rho_{0 \varepsilon}=\rho_{ \pm}$uniformly in $\varepsilon$. So for $\frac{\rho_{-}}{2}>0$, there exists $M_{1}>0$ such that

$$
\rho_{0 \varepsilon} \geq \frac{\rho_{-}}{2}, \quad \text { if } \quad|x| \geq M_{1} .
$$


Note that $\rho_{0 \varepsilon}$ has a lower bound in the domain $|x| \leq M_{1}$. Indeed, since $\left(\rho_{0}^{1}-\bar{\rho}_{0}\right) \in C(\mathbf{R})$, it follows that

$$
\left(\rho_{0}^{1}-\bar{\rho}_{0}\right) * j_{\varepsilon} \rightarrow \rho_{0}^{1}-\bar{\rho}_{0}, \quad \text { in } C\left(|x| \leq M_{1}\right)
$$

uniformly as $\varepsilon \rightarrow 0$. Hence

$$
\rho_{0 \varepsilon} \rightarrow \rho_{0}^{1}(x) \quad \text { in } C\left(|x| \leq M_{1}\right)
$$

uniformly as $\varepsilon \rightarrow 0$.

This implies that

$$
\rho_{0 \varepsilon}(x) \geq \frac{1}{2} \varepsilon^{\frac{1}{2 \alpha-2 \theta}} \quad \text { if } \quad|x| \leq M_{1} .
$$

Thus

$$
\rho_{0 \varepsilon}(x) \geq \min \left\{\frac{\rho_{-}}{2}, \frac{1}{2} \varepsilon^{\frac{1}{2 \alpha-2 \theta}}\right\}=\frac{1}{2} \varepsilon^{\frac{1}{2 \alpha-2 \theta}}, \quad \forall x \in \mathbf{R}
$$

for suitably small $\varepsilon$.

Moreover,

$$
\rho_{0 \varepsilon} \Psi\left(\rho_{0 \varepsilon}, \bar{\rho}_{0}\right) \rightarrow \rho_{0} \Psi\left(\rho_{0}, \bar{\rho}_{0}\right) \text { in } L^{1}(\mathbf{R}), \quad\left(\rho_{0 \varepsilon}^{\alpha-1 / 2}\right)_{x} \rightarrow\left(\rho_{0}^{\alpha-1 / 2}\right)_{x} \text { in } L^{2}(\mathbf{R}) .
$$

Therefore, it holds that

$$
\varepsilon^{2}\left[\left(\rho_{0 \varepsilon}^{\theta-\frac{1}{2}}\right)_{x}\right]^{2}=\left(\frac{\theta-\frac{1}{2}}{\alpha-\frac{1}{2}}\right)^{2} \varepsilon^{2} \rho_{0 \varepsilon}^{2 \theta-2 \alpha}\left[\left(\rho_{0 \varepsilon}^{\alpha-\frac{1}{2}}\right)_{x}\right]^{2} \leq C
$$

uniformly in $\varepsilon$.

To regularize $m_{0}$, one sets

$$
F_{0}=\rho_{0}\left(\frac{m_{0}}{\rho_{0}}-\bar{u}_{0}\right)^{3} \in L^{1}(\mathbf{R})
$$

and

$$
F_{0 \varepsilon}=F_{0} * j_{\varepsilon} .
$$

Then,

$$
F_{0 \varepsilon} \rightarrow F_{0}, \quad \text { a.e. }
$$

and

$$
F_{0 \varepsilon} \rightarrow F_{0}, \quad \text { in } L^{1}(\mathbf{R})
$$

Now we define

$$
m_{0 \varepsilon}=\rho_{0 \varepsilon}\left(\bar{u}_{0}+\left(\frac{F_{0 \varepsilon}}{\rho_{0 \varepsilon}}\right)^{\frac{1}{3}}\right) .
$$

Then

$$
\rho_{0 \varepsilon}\left(\frac{m_{0 \varepsilon}}{\rho_{0 \varepsilon}}-\bar{u}_{0}\right)^{3} \rightarrow \rho_{0}\left(\frac{m_{0}}{\rho_{0}}-\bar{u}_{0}\right)^{3} \text { in } L^{1}(\mathbf{R})
$$

and

$$
\rho_{0 \varepsilon}\left(\frac{m_{0 \varepsilon}}{\rho_{0 \varepsilon}}-\bar{u}_{0}\right)^{2} \rightarrow \rho_{0}\left(\frac{m_{0}}{\rho_{0}}-\bar{u}_{0}\right)^{2} \text { in } L^{1}(\mathbf{R}) .
$$

For any fixed $T>0$ and for any fixed $\varepsilon>0$, there exists a unique smooth approximate solution to (3.1) in the region $(x, t) \in \mathbf{R} \times(0, T)$ with initial data

$$
(\rho, \rho u)(0, x)=\left(\rho_{0 \varepsilon}, m_{0 \varepsilon}\right) .
$$


We refer to [26] for the wellposedness of the global strong solution to the approximate system (3.1) with (3.2).

The following estimates are crucial to prove our main results, which are based on the usual energy estimates and a new entropy estimates (see [1]-[4]).

Lemma 3.1 Let

$$
\frac{1}{2}<\alpha \leq \frac{\gamma+1}{2}
$$

Suppose that $\left(\rho_{\varepsilon}, u_{\varepsilon}\right)$ is a smooth solution to (3.1) satisfying $\rho_{\epsilon}>0$. Then for any $T>0$ and $\epsilon>0$ satisfying $\sqrt{\varepsilon} \ln (1+T) \leq \varepsilon^{\frac{1}{4}}$, the following estimates hold:

$$
\begin{aligned}
& \left.\sup _{t \in[0, T]} \int_{\mathbf{R}}\left\{\rho_{\varepsilon}\left(u_{\varepsilon}-\bar{u}\right)^{2}+\left[\left(\frac{\rho_{\varepsilon}^{\alpha-\frac{1}{2}}}{\alpha-\frac{1}{2}}\right)\right]_{x}\right]^{2}+\varepsilon^{2}\left[\left(\frac{\rho_{\varepsilon}^{\theta-\frac{1}{2}}}{\theta-\frac{1}{2}}\right)_{x}\right]^{2}+\rho_{\varepsilon} \Psi\left(\rho_{\varepsilon}, \bar{\rho}\right)\right\}(x, t) d x \\
& +\int_{0}^{T} \int_{\mathbf{R}}\left\{\bar{u}_{x}\left[p\left(\rho_{\varepsilon}\right)-p(\bar{\rho})-p^{\prime}(\bar{\rho})\left(\rho_{\varepsilon}-\bar{\rho}\right)\right]+\rho_{\varepsilon}\left(u_{\varepsilon}-\bar{u}\right)^{2} \bar{u}_{x}+\left(\rho_{\varepsilon}^{\alpha}+\varepsilon \rho_{\varepsilon}^{\theta}\right)\left[\left(u_{\varepsilon}-\bar{u}\right)_{x}\right]^{2}\right. \\
& \left.+\left[\left(\rho_{\varepsilon}^{\frac{\alpha+\gamma-1}{2}}-\bar{\rho}^{\frac{\alpha+\gamma-1}{2}}\right)_{x}\right]^{2}+\varepsilon\left[\left(\rho_{\varepsilon}^{\frac{\theta+\gamma-1}{2}}-\bar{\rho}^{\frac{\theta+\gamma-1}{2}}\right)_{x}\right]^{2}\right\}(x, t) d x d t \\
& \leq C,
\end{aligned}
$$

where $C$ is an universal constant independent of $\varepsilon$ and $T$.

In the following, the subscript $\varepsilon$ in the approximate solution $\left(\rho_{\varepsilon}, u_{\varepsilon}\right)(t, x)$ will be omitted for simplicity.

Proof: Step 1. Energy Equality

It follows from $(3.1)_{2}$ that

$$
\rho u_{t}+\rho u u_{x}+p(\rho)_{x}=\left(\mu_{\varepsilon}(\rho) u_{x}\right)_{x} .
$$

Subtracting (3.5) from the second equation of (2.4) gives

$\rho(u-\bar{u})_{t}+\rho u(u-\bar{u})_{x}+(p(\rho)-p(\bar{\rho}))_{x}+(\rho-\bar{\rho}) \bar{u}_{t}+(\rho u-\bar{\rho} \bar{u}) \bar{u}_{x}=\left(\mu_{\varepsilon}(\rho)(u-\bar{u})_{x}\right)_{x}+\left(\mu_{\varepsilon}(\rho) \bar{u}_{x}\right)_{x}$,

Multiplying (3.6) by $u-\bar{u}$, we get

$$
\begin{aligned}
& {\left[\frac{\rho(u-\bar{u})^{2}}{2}\right]_{t}+\left[\frac{\rho u(u-\bar{u})^{2}}{2}\right]_{x}+(u-\bar{u})(p(\rho)-p(\bar{\rho}))_{x}-\left[\mu_{\varepsilon}(\rho)(u-\bar{u})(u-\bar{u})_{x}\right]_{x}} \\
& +\mu_{\varepsilon}(\rho)\left[(u-\bar{u})_{x}\right]^{2}=\left[\mu_{\varepsilon}(\rho)\right]_{x} \bar{u}_{x}(u-\bar{u})+\mu_{\varepsilon}(\rho) \bar{u}_{x x}(u-\bar{u})-\left[(\rho-\bar{\rho}) \bar{u}_{t}+(\rho u-\bar{\rho} \bar{u}) \bar{u}_{x}\right](u-\bar{u}) .
\end{aligned}
$$

Note that $\Psi(\rho, \bar{\rho})$ defined in (2.5) satisfies

$$
\begin{aligned}
& {[\rho \Psi(\rho, \bar{\rho})]_{t}+[\rho u \Psi(\rho, \bar{\rho})]_{x}+(u-\bar{u})_{x}(p(\rho)-p(\bar{\rho}))+\bar{u}_{x}\left[\rho^{\gamma}-\bar{\rho}^{\gamma}-\gamma \bar{\rho}^{\gamma-1}(\rho-\bar{\rho})\right]} \\
& =-\frac{p(\bar{\rho})_{x}}{\bar{\rho}}(\rho-\bar{\rho})(u-\bar{u}) .
\end{aligned}
$$

It follows from (3.7) and (3.8) that

$$
\begin{aligned}
& {\left[\frac{\rho(u-\bar{u})^{2}}{2}+\rho \Psi(\rho, \bar{\rho})\right]_{t}+H_{1 x}(t, x)+\mu_{\varepsilon}(\rho)\left[(u-\bar{u})_{x}\right]^{2}+\bar{u}_{x}\left[\rho^{\gamma}-\bar{\rho}^{\gamma}-\gamma \bar{\rho}^{\gamma-1}(\rho-\bar{\rho})\right]} \\
& =\left[\mu_{\varepsilon}(\rho)\right]_{x} \bar{u}_{x}(u-\bar{u})+\mu_{\varepsilon}(\rho) \bar{u}_{x x}(u-\bar{u})-\left[(\rho-\bar{\rho}) \bar{u}_{t}+\left(\rho u-\bar{\rho} \bar{u} \bar{u}_{x}+\frac{p(\bar{\rho})_{x}}{\bar{\rho}}(\rho-\bar{\rho})\right](u-\bar{u}),\right.
\end{aligned}
$$


where

$$
H_{1}(t, x)=\frac{\rho u(u-\bar{u})^{2}}{2}+\rho u \Psi(\rho, \bar{\rho})+(u-\bar{u})(p(\rho)-p(\bar{\rho}))-\mu_{\varepsilon}(\rho)(u-\bar{u})(u-\bar{u})_{x} .
$$

Since

$$
(\rho-\bar{\rho}) \bar{u}_{t}+(\rho u-\bar{\rho} \bar{u}) \bar{u}_{x}+\frac{p(\bar{\rho})_{x}}{\bar{\rho}}(\rho-\bar{\rho})=\rho(u-\bar{u}) \bar{u}_{x}
$$

we obtain

$$
\begin{aligned}
& {\left[\frac{\rho(u-\bar{u})^{2}}{2}+\rho \Psi(\rho, \bar{\rho})\right]_{t}+H_{1 x}(t, x)+\mu_{\varepsilon}(\rho)\left[(u-\bar{u})_{x}\right]^{2}+\bar{u}_{x}\left[\rho^{\gamma}-\bar{\rho}^{\gamma}-\gamma \bar{\rho}^{\gamma-1}(\rho-\bar{\rho})\right]} \\
& +\rho(u-\bar{u})^{2} \bar{u}_{x}=\left[\mu_{\varepsilon}(\rho)\right]_{x} \bar{u}_{x}(u-\bar{u})+\mu_{\varepsilon}(\rho) \bar{u}_{x x}(u-\bar{u}) .
\end{aligned}
$$

Step 2. New Entropy Equality

Rewrite (3.6) as

$$
\rho(u-\bar{u})_{t}+\rho u(u-\bar{u})_{x}+(p(\rho)-p(\bar{\rho}))_{x}+(\rho-\bar{\rho}) \bar{u}_{t}+(\rho u-\bar{\rho} \bar{u}) \bar{u}_{x}=\left[\left(\rho^{\alpha-1}+\varepsilon \rho^{\theta-1}\right) \rho u_{x}\right]_{x} .
$$

Note that

$$
\left[\left(\rho^{\alpha-1}+\varepsilon \rho^{\theta-1}\right) \rho u_{x}\right]_{x}=-\rho\left(\varphi_{\varepsilon}^{\alpha, \theta}(\rho)\right)_{x t}-\rho u\left(\varphi_{\varepsilon}^{\alpha, \theta}(\rho)\right)_{x x},
$$

where $\varphi_{\varepsilon}^{\alpha, \theta}(\rho), 0<\theta<\frac{1}{2}$, is defined by

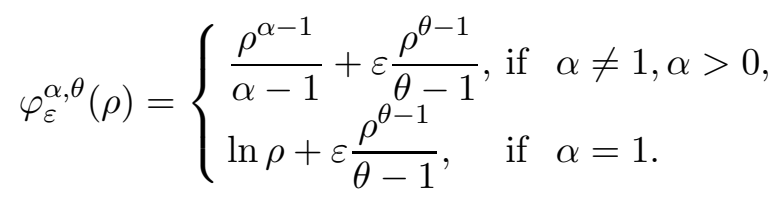

Thus (3.11) becomes

$\rho(u-\bar{u})_{t}+\rho u(u-\bar{u})_{x}+(p(\rho)-p(\bar{\rho}))_{x}+(\rho-\bar{\rho}) \bar{u}_{t}+(\rho u-\bar{\rho} \bar{u}) \bar{u}_{x}=-\rho\left(\varphi_{\varepsilon}^{\alpha, \theta}(\rho)\right)_{x t}-\rho u\left(\varphi_{\varepsilon}^{\alpha, \varepsilon}(\rho)\right)_{x x}$.

Multiplying (3.13) by $\left(\varphi_{\varepsilon}^{\alpha, \theta}(\rho)\right)_{x}$ shows that

$$
\begin{aligned}
& {\left[\frac{\rho\left(\varphi_{\varepsilon}^{\alpha, \theta}(\rho)\right)_{x}^{2}}{2}\right]_{t}+\left[\frac{\rho u\left(\varphi_{\varepsilon}^{\alpha, \theta}(\rho)\right)_{x}^{2}}{2}\right]_{x}+\left[\rho(u-\bar{u})\left(\varphi_{\varepsilon}^{\alpha, \theta}(\rho)\right)_{x}\right]_{t}+\left[\rho u(u-\bar{u})\left(\varphi_{\varepsilon}^{\alpha, \theta}(\rho)\right)_{x}\right]_{x}} \\
& -(u-\bar{u})\left[\rho\left(\varphi_{\varepsilon}^{\alpha, \theta}(\rho)\right)_{x t}+\rho u\left(\varphi_{\varepsilon}^{\alpha, \theta}(\rho)\right)_{x x}\right]+\left(\varphi_{\varepsilon}^{\alpha, \theta}(\rho)\right)_{x}(p(\rho)-p(\bar{\rho}))_{x} \\
& +\left(\varphi_{\varepsilon}^{\alpha, \theta}(\rho)\right)_{x}\left[(\rho-\bar{\rho}) \bar{u}_{t}+(\rho u-\bar{\rho} \bar{u}) \bar{u}_{x}\right]=0 .
\end{aligned}
$$

Combining (3.13) with (3.14) yields

$$
\begin{aligned}
& \left\{\frac{1}{2} \rho\left[(u-\bar{u})+\left(\varphi_{\varepsilon}^{\alpha, \theta}(\rho)\right)_{x}\right]^{2}\right\}_{t}+\left\{\frac{1}{2} \rho u\left[(u-\bar{u})+\left(\varphi_{\varepsilon}^{\alpha, \theta}(\rho)\right)_{x}\right]^{2}\right\}_{x}+(u-\bar{u})(p(\rho)-p(\bar{\rho}))_{x} \\
& +\left(\varphi_{\varepsilon}^{\alpha, \theta}(\rho)\right)_{x}(p(\rho)-p(\bar{\rho}))_{x}+(u-\bar{u})\left[(\rho-\bar{\rho}) \bar{u}_{t}+(\rho u-\bar{\rho} \bar{u}) \bar{u}_{x}\right] \\
& +\left(\varphi_{\varepsilon}^{\alpha, \theta}(\rho)\right)_{x}\left[(\rho-\bar{\rho}) \bar{u}_{t}+(\rho u-\bar{\rho} \bar{u}) \bar{u}_{x}\right]=0 .
\end{aligned}
$$


Step 3. A Priori Estimates

It follows from (3.8) and (3.15) that

$$
\begin{aligned}
& \left\{\frac{1}{2} \rho\left[(u-\bar{u})+\left(\varphi_{\varepsilon}^{\alpha, \theta}(\rho)\right)_{x}\right]^{2}+\rho \Psi(\rho, \bar{\rho})\right\}_{t}+\left\{\frac{1}{2} \rho u\left[(u-\bar{u})+\left(\varphi_{\varepsilon}^{\alpha, \theta}(\rho)\right)_{x}\right]^{2}+\rho u \Psi(\rho, \bar{\rho})\right. \\
& +(u-\bar{u})(p(\rho)-p(\bar{\rho}))\}_{x}+\bar{u}_{x}\left[p(\rho)-p(\bar{\rho})-p^{\prime}(\bar{\rho})(\rho-\bar{\rho})\right]+\rho(u-\bar{u})^{2} \bar{u}_{x} \\
& +\left(\varphi_{\varepsilon}^{\alpha, \theta}(\rho)\right)_{x}\left[(\rho-\bar{\rho}) \bar{u}_{t}+(\rho u-\bar{\rho} \bar{u}) \bar{u}_{x}+p(\rho)_{x}-p(\bar{\rho})_{x}\right]=0 .
\end{aligned}
$$

Now we deal with the last term on the left hand side of (3.16). Note that

$$
(\rho-\bar{\rho}) \bar{u}_{t}+(\rho u-\bar{\rho} \bar{u}) \bar{u}_{x}+p(\rho)_{x}-p(\bar{\rho})_{x}=\rho(u-\bar{u}) \bar{u}_{x}+\left[p(\rho)_{x}-\frac{\rho p(\bar{\rho})_{x}}{\bar{\rho}}\right],
$$

and

$$
\left(\varphi_{\varepsilon}^{\alpha, \theta}(\rho)\right)_{x}=\rho^{\alpha-2} \rho_{x}+\varepsilon \rho^{\theta-2} \rho_{x} .
$$

Thus

$$
\begin{aligned}
& \left(\varphi_{\varepsilon}^{\alpha, \theta}(\rho)\right)_{x}\left[(\rho-\bar{\rho}) \bar{u}_{t}+(\rho u-\bar{\rho} \bar{u}) \bar{u}_{x}+p(\rho)_{x}-p(\bar{\rho})_{x}\right] \\
& =\left(\frac{\rho^{\alpha}}{\alpha}+\varepsilon \frac{\rho^{\theta}}{\theta}\right)_{x}(u-\bar{u}) \bar{u}_{x}+\left(\rho^{\alpha-2} \rho_{x}+\varepsilon \rho^{\theta-2} \rho_{x}\right)\left[p(\rho)_{x}-\frac{\rho p(\bar{\rho})_{x}}{\bar{\rho}}\right] .
\end{aligned}
$$

Direct computations show

$$
\begin{aligned}
& \rho^{\alpha-2} \rho_{x}\left[p(\rho)_{x}-\frac{\rho p(\bar{\rho})_{x}}{\bar{\rho}}\right] \\
&=\frac{4 \gamma}{(\alpha+\gamma-1)^{2}}\left[\left(\rho^{\frac{\alpha+\gamma-1}{2}}-\bar{\rho}^{\frac{\alpha+\gamma-1}{2}}\right)_{x}\right]^{2}+\left[\frac{8 \gamma}{(\alpha+\gamma-1)^{2}}\left(\bar{\rho}^{\frac{\alpha+\gamma-1}{2}}\right)_{x}\left(\rho^{\frac{\alpha+\gamma-1}{2}}-\bar{\rho}^{\frac{\alpha+\gamma-1}{2}}\right)\right. \\
&\left.-\frac{2 \gamma}{\alpha(\alpha+\gamma-1)}\left(\bar{\rho}^{\frac{\alpha+\gamma-1}{2}}\right)_{x} \bar{\rho}^{\frac{\gamma-\alpha-1}{2}}\left(\rho^{\alpha}-\bar{\rho}^{\alpha}\right)\right]_{x}-\frac{8 \gamma}{(\alpha+\gamma-1)^{2}}\left(\bar{\rho}^{\frac{\alpha+\gamma-1}{2}}\right)_{x x}\left(\rho^{\frac{\alpha+\gamma-1}{2}}-\bar{\rho}^{\frac{\alpha+\gamma-1}{2}}\right) \\
&+\frac{2 \gamma}{\alpha(\alpha+\gamma-1)}\left[\left(\bar{\rho}^{\frac{\alpha+\gamma-1}{2}}\right)_{x} \bar{\rho}^{\frac{\gamma-\alpha-1}{2}}\right]_{x}\left(\rho^{\alpha}-\bar{\rho}^{\alpha}\right) .
\end{aligned}
$$

and

$$
\begin{aligned}
& \rho^{\theta-2} \rho_{x}\left[p(\rho)_{x}-\frac{\rho p(\bar{\rho})_{x}}{\bar{\rho}}\right] \\
& =\frac{4 \gamma}{(\theta+\gamma-1)^{2}}\left[\left(\rho^{\frac{\theta+\gamma-1}{2}}-\bar{\rho}^{\frac{\theta+\gamma-1}{2}}\right)_{x}\right]^{2}+\left[\frac{8 \gamma}{(\theta+\gamma-1)^{2}}\left(\bar{\rho}^{\frac{\theta+\gamma-1}{2}}\right)_{x}\left(\rho^{\frac{\theta+\gamma-1}{2}}-\bar{\rho}^{\frac{\theta+\gamma-1}{2}}\right)\right. \\
& \left.\quad-\frac{2 \gamma}{\theta(\theta+\gamma-1)}\left(\bar{\rho}^{\frac{\theta+\gamma-1}{2}}\right)_{x} \bar{\rho}^{\frac{\gamma-\theta-1}{2}}\left(\rho^{\theta}-\bar{\rho}^{\theta}\right)\right]_{x}-\frac{8 \gamma}{(\theta+\gamma-1)^{2}}\left(\bar{\rho}^{\frac{\theta+\gamma-1}{2}}\right)_{x x}\left(\rho^{\frac{\theta+\gamma-1}{2}}-\bar{\rho}^{\frac{\theta+\gamma-1}{2}}\right) \\
& \quad+\frac{2 \gamma}{\theta(\theta+\gamma-1)}\left[\left(\bar{\rho}^{\frac{\theta+\gamma-1}{2}}\right)_{x} \bar{\rho}^{\frac{\gamma-\theta-1}{2}}\right]_{x}\left(\rho^{\theta}-\bar{\rho}^{\theta}\right) .
\end{aligned}
$$


Substituting (3.19)-(3.21) into (3.16) gives

$$
\begin{aligned}
& \left\{\frac{1}{2} \rho\left[(u-\bar{u})+\left(\varphi_{\varepsilon}^{\alpha, \theta}(\rho)\right)_{x}\right]^{2}+\rho \Psi(\rho, \bar{\rho})\right\}_{t}+H_{2 x}(t, x)+\bar{u}_{x}\left[p(\rho)-p(\bar{\rho})-p^{\prime}(\bar{\rho})(\rho-\bar{\rho})\right] \\
& +\rho(u-\bar{u})^{2} \bar{u}_{x}+\left(\frac{\rho^{\alpha}}{\alpha}+\varepsilon \frac{\rho^{\theta}}{\theta}\right)_{x}(u-\bar{u}) \bar{u}_{x}+\frac{4 \gamma}{(\alpha+\gamma-1)^{2}}\left[\left(\rho^{\frac{\alpha+\gamma-1}{2}}-\bar{\rho}^{\frac{\alpha+\gamma-1}{2}}\right)_{x}\right]^{2} \\
& +\varepsilon \frac{4 \gamma}{(\theta+\gamma-1)^{2}}\left[\left(\rho^{\frac{\theta+\gamma-1}{2}}-\bar{\rho}^{\frac{\theta+\gamma-1}{2}}\right)_{x}\right]^{2}=\frac{8 \gamma}{(\alpha+\gamma-1)^{2}}\left(\bar{\rho}^{\frac{\alpha+\gamma-1}{2}}\right)_{x x}\left(\rho^{\frac{\alpha+\gamma-1}{2}}-\bar{\rho}^{\frac{\alpha+\gamma-1}{2}}\right) \\
& +\varepsilon \frac{8 \gamma}{(\theta+\gamma-1)^{2}}\left(\bar{\rho}^{\frac{\theta+\gamma-1}{2}}\right)_{x x}\left(\rho^{\frac{\theta+\gamma-1}{2}}-\bar{\rho}^{\frac{\theta+\gamma-1}{2}}\right)-\frac{2 \gamma}{\alpha(\alpha+\gamma-1)}\left[\left(\bar{\rho}^{\frac{\alpha+\gamma-1}{2}}\right)_{x} \bar{\rho}^{\frac{\gamma-\alpha-1}{2}}\right]_{x}\left(\rho^{\alpha}-\bar{\rho}^{\alpha}\right) \\
& -\varepsilon \frac{2 \gamma}{\theta(\theta+\gamma-1)}\left[\left(\bar{\rho}^{\frac{\theta+\gamma-1}{2}}\right)_{x} \bar{\rho}^{\frac{\gamma-\theta-1}{2}}\right]_{x}\left(\rho^{\theta}-\bar{\rho}^{\theta}\right),
\end{aligned}
$$

where

$$
\begin{aligned}
H_{2}(t, x)= & \frac{1}{2} \rho u\left[(u-\bar{u})+\left(\varphi_{\varepsilon}^{\alpha, \theta}(\rho)\right)_{x}\right]^{2}+\rho u \Psi(\rho, \bar{\rho})+(u-\bar{u})(p(\rho)-p(\bar{\rho})) \\
& +\frac{8 \gamma}{(\alpha+\gamma-1)^{2}}\left(\bar{\rho}^{\frac{\alpha+\gamma-1}{2}}\right)_{x}\left(\rho^{\frac{\alpha+\gamma-1}{2}}-\bar{\rho}^{\frac{\alpha+\gamma-1}{2}}\right) \\
& -\frac{2 \gamma}{\alpha(\alpha+\gamma-1)}\left(\bar{\rho}^{\frac{\alpha+\gamma-1}{2}}\right)_{x} \bar{\rho}^{\frac{\gamma-\alpha-1}{2}}\left(\rho^{\alpha}-\bar{\rho}^{\alpha}\right) \\
& +\varepsilon \frac{8 \gamma}{(\theta+\gamma-1)^{2}}\left(\bar{\rho}^{\frac{\theta+\gamma-1}{2}}\right)_{x}\left(\rho^{\frac{\theta+\gamma-1}{2}}-\bar{\rho}^{\frac{\theta+\gamma-1}{2}}\right) \\
& -\varepsilon \frac{2 \gamma}{\theta(\theta+\gamma-1)}\left(\bar{\rho}^{\frac{\theta+\gamma-1}{2}}\right)_{x} \bar{\rho}^{\frac{\gamma-\theta-1}{2}}\left(\rho^{\theta}-\bar{\rho}^{\theta}\right)
\end{aligned}
$$

Multiplying (3.22) by $\alpha$ and then adding up to (3.10) and noticing that $\left[\mu_{\varepsilon}(\rho)\right]_{x}=\left(\rho^{\alpha}\right)_{x}+\varepsilon\left(\rho^{\theta}\right)_{x}$ in the right hand side of (3.10), we get

$$
\begin{aligned}
& \left\{\frac{\alpha}{2} \rho\left[(u-\bar{u})+\left(\varphi_{\varepsilon}^{\alpha, \theta}(\rho)\right)_{x}\right]^{2}+\frac{\rho(u-\bar{u})^{2}}{2}+(\alpha+1) \rho \Psi(\rho, \bar{\rho})\right\}_{t}+\left[\alpha H_{2}(t, x)+H_{1}(t, x)\right]_{x} \\
& +(\alpha+1) \bar{u}_{x}\left[p(\rho)-p(\bar{\rho})-p^{\prime}(\bar{\rho})(\rho-\bar{\rho})\right]+(\alpha+1) \rho(u-\bar{u})^{2} \bar{u}_{x}+\left(\rho^{\alpha}+\varepsilon \rho^{\theta}\right)\left[(u-\bar{u})_{x}\right]^{2} \\
& +\frac{4 \alpha \gamma}{(\alpha+\gamma-1)^{2}}\left[\left(\rho^{\frac{\alpha+\gamma-1}{2}}-\bar{\rho}^{\frac{\alpha+\gamma-1}{2}}\right)_{x}\right]^{2}+\varepsilon \frac{4 \alpha \gamma}{(\theta+\gamma-1)^{2}}\left[\left(\rho^{\frac{\theta+\gamma-1}{2}}-\bar{\rho}^{\frac{\theta+\gamma-1}{2}}\right)_{x}\right]^{2} \\
& =\rho^{\alpha} \bar{u}_{x x}(u-\bar{u})+\varepsilon\left[\rho^{\theta} \bar{u}_{x x}(u-\bar{u})+\left(1-\frac{\alpha}{\theta}\right)\left(\rho^{\theta}\right)_{x}(u-\bar{u}) \bar{u}_{x}\right] \\
& +\frac{8 \alpha \gamma}{(\alpha+\gamma-1)^{2}}\left(\bar{\rho}^{\frac{\alpha+\gamma-1}{2}}\right)_{x x}\left(\rho^{\frac{\alpha+\gamma-1}{2}}-\bar{\rho}^{\frac{\alpha+\gamma-1}{2}}\right) \\
& +\varepsilon \frac{8 \alpha \gamma}{(\theta+\gamma-1)^{2}}\left(\bar{\rho}^{\frac{\theta+\gamma-1}{2}}\right)_{x x}\left(\rho^{\frac{\theta+\gamma-1}{2}}-\bar{\rho}^{\frac{\theta+\gamma-1}{2}}\right)-\frac{2 \gamma}{(\alpha+\gamma-1)}\left[\left(\bar{\rho}^{\frac{\alpha+\gamma-1}{2}}\right)_{x} \bar{\rho}^{\frac{\gamma-\alpha-1}{2}}\right]_{x}\left(\rho^{\alpha}-\bar{\rho}^{\alpha}\right) \\
& -\varepsilon \frac{2 \alpha \gamma}{\theta(\theta+\gamma-1)}\left[\left(\bar{\rho}^{\frac{\theta+\gamma-1}{2}}\right)_{x} \bar{\rho}^{\frac{\gamma-\theta-1}{2}}\right]_{x}\left(\rho^{\theta}-\bar{\rho}^{\theta}\right) \\
& :=\sum_{i=1}^{6} I_{i} .
\end{aligned}
$$


Integrating (3.24) over $[0, t] \times \mathbf{R}$ with respect to $t, x$ gives

$$
\begin{aligned}
& \int_{\mathbf{R}}\left\{\frac{\alpha}{2} \rho\left[(u-\bar{u})+\left(\varphi_{\varepsilon}^{\alpha, \theta}(\rho)\right)_{x}\right]^{2}+\frac{\rho(u-\bar{u})^{2}}{2}+(\alpha+1) \rho \Psi(\rho, \bar{\rho})\right\}(t, x) d x \\
& +\int_{0}^{t} \int_{\mathbf{R}}\left\{(\alpha+1) \bar{u}_{x}\left[p(\rho)-p(\bar{\rho})-p^{\prime}(\bar{\rho})(\rho-\bar{\rho})\right]+(\alpha+1) \rho(u-\bar{u})^{2} \bar{u}_{x}\right. \\
& +\left(\rho^{\alpha}+\varepsilon \rho^{\theta}\right)\left[(u-\bar{u})_{x}\right]^{2}+\frac{4 \alpha \gamma}{(\alpha+\gamma-1)^{2}}\left[\left(\rho^{\frac{\alpha+\gamma-1}{2}}-\bar{\rho}^{\frac{\alpha+\gamma-1}{2}}\right)_{x}\right]^{2} \\
& \left.+\varepsilon \frac{4 \alpha \gamma}{(\theta+\gamma-1)^{2}}\left[\left(\rho^{\frac{\theta+\gamma-1}{2}}-\bar{\rho}^{\frac{\theta+\gamma-1}{2}}\right)_{x}\right]^{2}\right\} d x d \tau \\
& =\int_{0}^{t} \int_{\mathbf{R}} \sum_{i=1}^{6} I_{i} d x d \tau .
\end{aligned}
$$

We now estimate the right hand side of (3.25) terms by terms. First,

$$
\begin{aligned}
\int_{0}^{t} \int_{\mathbf{R}} I_{1} d x d \tau & =\int_{0}^{t} \int_{\mathbf{R}} \rho^{\alpha} \bar{u}_{x x}(u-\bar{u}) d x d \tau \\
& =\int_{0}^{t} \int_{\mathbf{R}} \sqrt{\rho}(u-\bar{u}) \rho^{\alpha-\frac{1}{2}} \bar{u}_{x x} d x d \tau \\
& =\int_{0}^{t} \int_{\mathbf{R}} \sqrt{\rho}(u-\bar{u}) \rho^{\alpha-\frac{1}{2}} \bar{u}_{x x}\left[\left.\mathbf{1}\right|_{\left\{0 \leq \rho \leq 2 \rho_{+}\right\}}+\left.\mathbf{1}\right|_{\left\{\rho \geq 2 \rho_{+}\right\}}\right] d x d \tau \\
& :=J_{1}^{1}+J_{1}^{2},
\end{aligned}
$$

where $\left.\mathbf{1}\right|_{\Omega}$ is the characteristic function of a set $\Omega \subset(0, t) \times \mathbf{R}$.

Using Lemma 2.2 (and its Remark), noting that $\alpha>\frac{1}{2}$, we have

$$
\begin{aligned}
J_{1}^{1} & \leq C \int_{0}^{t}\|\sqrt{\rho}(u-\bar{u})\|_{L^{2}(\mathbf{R})}\left\|\bar{u}_{x x}\right\|_{L^{2}(\mathbf{R})} d \tau \\
& \leq C \sup _{t \in[0, T]}\|\sqrt{\rho}(u-\bar{u})\|_{L^{2}(\mathbf{R})} \int_{0}^{t}\left\|\bar{u}_{x x}\right\|_{L^{2}(\mathbf{R})} d \tau \\
& \leq C \sup _{t \in[0, T]}\|\sqrt{\rho}(u-\bar{u})\|_{L^{2}(\mathbf{R})},
\end{aligned}
$$

and

$$
\begin{aligned}
J_{1}^{2}= & \left.\int_{0}^{t} \int_{\mathbf{R}} \sqrt{\rho}(u-\bar{u})\left(\rho^{\alpha-\frac{1}{2}}-\bar{\rho}^{\alpha-\frac{1}{2}}\right) \bar{u}_{x x} \mathbf{1}\right|_{\left\{\rho \geq 2 \rho_{+}\right\}} d x d \tau+\left.\int_{0}^{t} \int_{\mathbf{R}} \sqrt{\rho}(u-\bar{u}) \bar{\rho}^{\alpha-\frac{1}{2}} \bar{u}_{x x} \mathbf{1}\right|_{\{\rho \geq 2 \rho+\}} d x d \tau \\
\leq & C \sup _{t \in[0, T]}\|\sqrt{\rho}(u-\bar{u})\|_{L^{2}(\mathbf{R})} \sup _{t \in[0, T]}\left\|\left.\left(\rho^{\alpha-\frac{1}{2}}-\bar{\rho}^{\alpha-\frac{1}{2}}\right) \mathbf{1}\right|_{\left\{\rho \geq 2 \rho_{+}\right\}}\right\|_{L^{2}(\mathbf{R})} \int_{0}^{t}\left\|\bar{u}_{x x}\right\|_{L^{\infty}(\mathbf{R})} d \tau \\
& +C \sup _{t \in[0, T]}\|\sqrt{\rho}(u-\bar{u})\|_{L^{2}(\mathbf{R})} \int_{0}^{t}\left\|\bar{u}_{x x}\right\|_{L^{2}(\mathbf{R})} d \tau \\
\leq & C \eta^{\frac{2}{4 q+1}} \sup _{t \in[0, T]}\|\sqrt{\rho}(u-\bar{u})\|_{L^{2}(\mathbf{R})} \sup _{t \in[0, T]}\left\|\left.\left(\rho^{\alpha-\frac{1}{2}}-\bar{\rho}^{\alpha-\frac{1}{2}}\right) \mathbf{1}\right|_{\left\{\rho \geq 2 \rho_{+}\right\}}\right\|_{L^{2}(\mathbf{R})} \int_{0}^{t}(1+\tau)^{-1-\frac{1}{4 q+1}} d \tau \\
& +C \sup _{t \in[0, T]}\|\sqrt{\rho}(u-\bar{u})\|_{L^{2}(\mathbf{R})} \\
\leq & C \eta^{\frac{2}{4 q+1}}\left[\sup _{t \in[0, T]}\|\sqrt{\rho}(u-\bar{u})\|_{L^{2}(\mathbf{R})}^{2}+\sup _{t \in[0, T]}\left\|\left.\left(\rho^{\alpha-\frac{1}{2}}-\bar{\rho}^{\alpha-\frac{1}{2}}\right) \mathbf{1}\right|_{\left\{\rho \geq 2 \rho_{+}\right\}}\right\|_{L^{2}(\mathbf{R})}^{2}\right]+C_{\eta} .
\end{aligned}
$$


Note that if $\alpha$ and $\gamma$ satisfy

$$
0<2\left(\alpha-\frac{1}{2}\right) \leq \gamma, \quad \text { i.e., } \quad \frac{1}{2}<\alpha \leq \frac{\gamma+1}{2},
$$

then

$$
\begin{aligned}
\lim _{\rho \rightarrow+\infty} \frac{\left(\rho^{\alpha-\frac{1}{2}}-\bar{\rho}^{\alpha-\frac{1}{2}}\right)^{2}}{\rho \Psi(\rho, \bar{\rho})} & =\lim _{\rho \rightarrow+\infty} \frac{(\gamma-1)\left(\rho^{\alpha-\frac{1}{2}}-\bar{\rho}^{\alpha-\frac{1}{2}}\right)^{2}}{\rho^{\gamma}-\bar{\rho}^{\gamma}-\gamma \bar{\rho}^{\gamma-1}(\rho-\bar{\rho})} \\
& \leq C .
\end{aligned}
$$

Thus if $\frac{1}{2}<\alpha \leq \frac{\gamma+1}{2}$, then

$$
\sup _{t \in[0, T]}\left\|\left.\left(\rho^{\alpha-\frac{1}{2}}-\bar{\rho}^{\alpha-\frac{1}{2}}\right) \mathbf{1}\right|_{\left\{\rho \geq 2 \rho_{+}\right\}}\right\|_{L^{2}(\mathbf{R})}^{2} \leq C \sup _{t \in[0, T]}\|\rho \Psi(\rho, \bar{\rho})\|_{L^{1}(\mathbf{R})}
$$

for some uniform constant $C>0$.

Combining (3.27)-(3.31) together shows that

$$
\int_{0}^{t} \int_{\mathbf{R}} I_{1} d x d \tau \leq C \eta^{\frac{2}{4 q+1}}\left[\sup _{t \in[0, T]}\|\sqrt{\rho}(u-\bar{u})\|_{L^{2}(\mathbf{R})}^{2}+\sup _{t \in[0, T]}\|\rho \Psi(\rho, \bar{\rho})\|_{L^{1}(\mathbf{R})}\right]+C_{\eta} .
$$

Next, we estimate $\int_{0}^{t} \int_{\mathbf{R}} I_{2} d x d \tau$ which can be rewritten as

$$
\begin{aligned}
\int_{0}^{t} \int_{\mathbf{R}} I_{2} d x d \tau & =\varepsilon \int_{0}^{t} \int_{\mathbf{R}}\left[\rho^{\theta} \bar{u}_{x x}(u-\bar{u})+\left(1-\frac{\alpha}{\theta}\right)\left(\rho^{\theta}\right)_{x}(u-\bar{u}) \bar{u}_{x}\right] d x d \tau \\
& =-\varepsilon \int_{0}^{t} \int_{\mathbf{R}}\left[\rho^{\theta} \bar{u}_{x}(u-\bar{u})_{x}+\frac{\alpha}{\theta}\left(\rho^{\theta}\right)_{x}(u-\bar{u}) \bar{u}_{x}\right] d x d \tau \\
& :=J_{2}^{1}+J_{2}^{2}
\end{aligned}
$$

Using Young inequality, one has

$$
\begin{aligned}
J_{2}^{1} & =-\varepsilon \int_{0}^{t} \int_{\mathbf{R}} \rho^{\theta} \bar{u}_{x}(u-\bar{u})_{x} d x d \tau \\
& \leq \frac{\varepsilon}{4} \int_{0}^{t} \int_{\mathbf{R}} \rho^{\theta}\left[(u-\bar{u})_{x}\right]^{2} d x d \tau+\varepsilon \int_{0}^{t} \int_{\mathbf{R}} \rho^{\theta} \bar{u}_{x}^{2} d x d \tau .
\end{aligned}
$$

By Lemma 2.2, one can obtain

$$
\begin{aligned}
\varepsilon \int_{0}^{t} \int_{\mathbf{R}} \rho^{\theta} \bar{u}_{x}^{2} d x d \tau & =\varepsilon \int_{0}^{t} \int_{\mathbf{R}} \rho^{\theta}\left(\mathbf{1}_{\left\{0 \leq \rho \leq 2 \rho_{+}\right\}}+\mathbf{1}_{\left\{\rho \geq 2 \rho_{+}\right\}}\right) \bar{u}_{x}^{2} d x d \tau \\
& \leq C \varepsilon \int_{0}^{t} \int_{\mathbf{R}} \bar{u}_{x}^{2} d x d \tau+\varepsilon \int_{0}^{t} \int_{\mathbf{R}}\left(\rho^{\theta}-\bar{\rho}^{\theta}\right) \mathbf{1}_{\left\{\rho \geq 2 \rho_{+}\right\}} \bar{u}_{x}^{2} d x d \tau \\
& \leq C \varepsilon \ln (1+T)+C \varepsilon \sup _{t \in[0, T]}\left\|\left(\rho^{\theta}-\bar{\rho}^{\theta}\right) \mathbf{1}_{\left\{\rho \geq 2 \rho_{+}\right\}}\right\|_{L^{1}(\mathbf{R})} \int_{0}^{t}\left\|\bar{u}_{x}\right\|_{L^{\infty}(\mathbf{R})}^{2} d \tau \\
& \leq C \varepsilon \ln (1+T)+C \varepsilon \sup _{t \in[0, T]}\|\rho \Psi(\rho, \bar{\rho})\|_{L^{1}(\mathbf{R})}
\end{aligned}
$$

due to the fact that

$$
\lim _{\rho \rightarrow+\infty} \frac{\left|\left(\rho^{\theta}-\bar{\rho}^{\theta}\right)\right| \mathbf{1}_{\left\{\rho \geq 2 \rho_{+}\right\}}}{\rho \Psi(\rho, \bar{\rho})}=0
$$


Moreover, direct estimates show

$$
\begin{aligned}
J_{2}^{2} & =-\frac{\varepsilon \theta}{\theta-\frac{1}{2}} \int_{0}^{t} \int_{\mathbf{R}}\left(\rho^{\theta-\frac{1}{2}}\right)_{x} \bar{u}_{x} \sqrt{\rho}(u-\bar{u}) d x d \tau \\
& \leq C \sqrt{\varepsilon} \sup _{t \in[0, T]}\left\|\sqrt{\varepsilon}\left(\rho^{\theta-\frac{1}{2}}\right)_{x}\right\|_{L^{2}(\mathbf{R})} \sup _{t \in[0, T]}\|\sqrt{\rho}(u-\bar{u})\|_{L^{2}(\mathbf{R})} \int_{0}^{t}\left\|\bar{u}_{x}\right\|_{L^{\infty}(\mathbf{R})} d \tau \\
& \leq C \sqrt{\varepsilon} \ln (1+T)\left[\sup _{t \in[0, T]}\left\|\sqrt{\varepsilon}\left(\rho^{\theta-\frac{1}{2}}\right)_{x}\right\|_{L^{2}(\mathbf{R})}^{2}+\sup _{t \in[0, T]}\|\sqrt{\rho}(u-\bar{u})\|_{L^{2}(\mathbf{R})}^{2}\right] .
\end{aligned}
$$

Thus the term $I_{2}$ is estimated as

$$
\begin{aligned}
\int_{0}^{t} \int_{\mathbf{R}} I_{2} d x d \tau & \leq C \sqrt{\varepsilon} \ln (1+T)\left[1+\sup _{t \in[0, T]}\left\|\sqrt{\varepsilon}\left(\rho^{\theta-\frac{1}{2}}\right) x\right\|_{L^{2}(\mathbf{R})}^{2}+\sup _{t \in[0, T]}\|\sqrt{\rho}(u-\bar{u})\|_{L^{2}(\mathbf{R})}^{2}\right] \\
& +C \varepsilon \sup _{t \in[0, T]}\|\rho \Psi(\rho, \bar{\rho})\|_{L^{1}(\mathbf{R})} .
\end{aligned}
$$

The term $I_{3}$ can be estimated as follows.

$$
\begin{aligned}
\int_{0}^{t} \int_{\mathbf{R}} I_{3} d x d \tau & =\int_{0}^{t} \int_{\mathbf{R}} I_{3}\left(\mathbf{1}_{\left\{|\rho-\bar{\rho}| \leq \frac{\rho_{-}}{2}\right\}}+\mathbf{1}_{\left\{|\rho-\bar{\rho}|>\frac{\rho_{-}}{2}\right\}}\right) d x d \tau \\
& :=J_{3}^{1}+J_{3}^{2} .
\end{aligned}
$$

Direct estimates lead to

$$
\begin{aligned}
J_{3}^{1} & =\int_{0}^{t} \int_{\mathbf{R}} \frac{8 \alpha \gamma}{(\alpha+\gamma-1)^{2}}\left(\bar{\rho}^{\frac{\alpha+\gamma-1}{2}}\right)_{x x}\left(\rho^{\frac{\alpha+\gamma-1}{2}}-\bar{\rho}^{\frac{\alpha+\gamma-1}{2}}\right) \mathbf{1}_{\left\{|\rho-\bar{\rho}| \leq \frac{\rho_{-}}{2}\right\}} d x d \tau \\
& \leq C \sup _{t \in[0, T]}\left\|\left(\rho^{\frac{\alpha+\gamma-1}{2}}-\bar{\rho}^{\frac{\alpha+\gamma-1}{2}}\right) \mathbf{1}_{\left\{|\rho-\bar{\rho}| \leq \frac{\rho_{-}}{2}\right\}}\right\|_{L^{2}(\mathbf{R})} \int_{0}^{t}\left\|\left(\bar{\rho}^{\frac{\alpha+\gamma-1}{2}}\right)_{x x}\right\|_{L^{2}(\mathbf{R})} d \tau \\
& \leq C\|\sqrt{\rho \Psi(\rho, \bar{\rho})}\|_{L^{2}(\mathbf{R})},
\end{aligned}
$$

where one has used the fact that

$$
\left\|\left(\rho^{\frac{\alpha+\gamma-1}{2}}-\bar{\rho}^{\frac{\alpha+\gamma-1}{2}}\right) \mathbf{1}_{\left\{|\rho-\bar{\rho}| \leq \frac{\rho_{-}}{2}\right\}}\right\|_{L^{2}(\mathbf{R})} \leq C\|\sqrt{\rho \Psi(\rho, \bar{\rho})}\|_{L^{2}(\mathbf{R})} .
$$

Moreover, due to the facts that

$$
\lim _{\rho \rightarrow 0+} \frac{\left|\rho^{\frac{\alpha+\gamma-1}{2}}-\bar{\rho}^{\frac{\alpha+\gamma-1}{2}}\right| \mathbf{1}_{\left\{|\rho-\bar{\rho}|>\frac{\bar{\rho}}{2}\right\}}}{\rho \Psi(\rho, \bar{\rho})}=\bar{\rho}^{\frac{\alpha-\gamma-1}{2}} \leq C,
$$

and for

$$
\begin{gathered}
\frac{\alpha+\gamma-1}{2} \leq \gamma, \quad \text { i. e., } \quad \alpha \leq \gamma+1, \\
\lim _{\rho \rightarrow+\infty} \frac{\left|\rho^{\frac{\alpha+\gamma-1}{2}}-\bar{\rho}^{\frac{\alpha+\gamma-1}{2}}\right| \mathbf{1}_{\left\{|\rho-\bar{\rho}|>\frac{\rho_{-}}{2}\right\}}}{\rho \Psi(\rho, \bar{\rho})} \leq C,
\end{gathered}
$$


we can estimate $J_{3}^{2}$ as

$$
\begin{aligned}
J_{3}^{2} & =\int_{0}^{t} \int_{\mathbf{R}} \frac{8 \alpha \gamma}{(\alpha+\gamma-1)^{2}}\left(\bar{\rho}^{\frac{\alpha+\gamma-1}{2}}\right)_{x x}\left(\rho^{\frac{\alpha+\gamma-1}{2}}-\bar{\rho}^{\frac{\alpha+\gamma-1}{2}}\right) \mathbf{1}_{\left\{|\rho-\bar{\rho}|>\frac{\rho-}{2}\right\}} d x d \tau \\
& \leq C \sup _{t \in[0, T]}\left\|\left(\rho^{\frac{\alpha+\gamma-1}{2}}-\bar{\rho}^{\frac{\alpha+\gamma-1}{2}}\right) \mathbf{1}_{\left\{|\rho-\bar{\rho}|>\frac{\rho_{-}}{2}\right\}}\right\|_{L^{1}(\mathbf{R})} \int_{0}^{t}\left\|\left(\bar{\rho}^{\frac{\alpha+\gamma-1}{2}}\right)_{x x}\right\|_{L^{\infty}(\mathbf{R})} d \tau \\
& \leq C \eta^{\frac{2}{4 q+1}}\|\rho \Psi(\rho, \bar{\rho})\|_{L^{1}(\mathbf{R})} .
\end{aligned}
$$

The term $I_{4}$ can be handled similarly because $0<\theta<\frac{1}{2}<\alpha \leq \gamma+1$. Now we turn to

$$
\begin{aligned}
\int_{0}^{t} \int_{\mathbf{R}} I_{5} d x d \tau & =\int_{0}^{t} \int_{\mathbf{R}} I_{5}\left(\mathbf{1}_{\left\{|\rho-\bar{\rho}| \leq \frac{\rho_{-}}{2}\right\}}+\mathbf{1}_{\left\{|\rho-\bar{\rho}|>\frac{\rho_{-}}{2}\right\}}\right) d x d \tau \\
& :=J_{5}^{1}+J_{5}^{2} .
\end{aligned}
$$

One has

$$
\begin{aligned}
J_{5}^{1} & =\int_{0}^{t} \int_{\mathbf{R}} \frac{2 \gamma}{(\alpha+\gamma-1)}\left[\left(\bar{\rho}^{\frac{\alpha+\gamma-1}{2}}\right)_{x} \bar{\rho}^{\frac{\gamma-\alpha-1}{2}}\right]_{x}\left(\rho^{\alpha}-\bar{\rho}^{\alpha}\right) \mathbf{1}_{\left\{|\rho-\bar{\rho}| \leq \frac{\rho-}{2}\right\}} d x d \tau \\
& \leq C \sup _{t \in[0, T]}\left\|\left(\rho^{\alpha}-\bar{\rho}^{\alpha}\right) \mathbf{1}_{\left\{|\rho-\bar{\rho}| \leq \frac{\rho_{-}}{2}\right\}}\right\|_{L^{2}(\mathbf{R})} \int_{0}^{t}\left\|\left[\left(\bar{\rho}^{\frac{\alpha+\gamma-1}{2}}\right)_{x} \bar{\rho}^{\frac{\gamma-\alpha-1}{2}}\right]_{x}\right\|_{L^{2}(\mathbf{R})} d \tau \\
& \leq C \sup _{t \in[0, T]}\|\sqrt{\rho \Psi(\rho, \bar{\rho})}\|_{L^{2}(\mathbf{R})},
\end{aligned}
$$

and

$$
\begin{aligned}
J_{5}^{2} & =\int_{0}^{t} \int_{\mathbf{R}} \frac{2 \gamma}{(\alpha+\gamma-1)}\left[\left(\bar{\rho}^{\frac{\alpha+\gamma-1}{2}}\right)_{x} \bar{\rho}^{\frac{\gamma-\alpha-1}{2}}\right]_{x}\left(\rho^{\alpha}-\bar{\rho}^{\alpha}\right) \mathbf{1}_{\left\{|\rho-\bar{\rho}|>\frac{\rho_{-}}{2}\right\}} d x d \tau \\
& \leq C \sup _{t \in[0, T]}\left\|\left(\rho^{\alpha}-\bar{\rho}^{\alpha}\right) \mathbf{1}_{\left\{|\rho-\bar{\rho}|>\frac{\rho_{-}}{2}\right\}}\right\|_{L^{1}(\mathbf{R})} \int_{0}^{t}\left\|\left[\left(\bar{\rho}^{\frac{\alpha+\gamma-1}{2}}\right)_{x} \bar{\rho}^{\frac{\gamma-\alpha-1}{2}}\right]_{x}\right\|_{L^{\infty}(\mathbf{R})} d \tau \\
& \leq C \eta^{\frac{2}{4 q+1}} \sup _{t \in[0, T]}\|\sqrt{\rho \Psi(\rho, \bar{\rho})}\|_{L^{1}(\mathbf{R})},
\end{aligned}
$$

where the following facts have been used:

$$
\begin{gathered}
\lim _{\rho \rightarrow 0+} \frac{\left|\rho^{\alpha}-\bar{\rho}^{\alpha}\right| \mathbf{1}_{\left\{|\rho-\bar{\rho}|>\frac{\rho_{-}}{2}\right\}}}{\rho \Psi(\rho, \bar{\rho})}=\bar{\rho}^{\alpha-\gamma} \leq C, \\
\lim _{\rho \rightarrow+\infty} \frac{\left|\rho^{\alpha}-\bar{\rho}^{\alpha}\right| \mathbf{1}_{\left\{|\rho-\bar{\rho}|>\frac{\rho_{-}}{2}\right\}}}{\rho \Psi(\rho, \bar{\rho})} \leq C, \quad \text { for } \quad \frac{1}{2}<\alpha \leq \gamma .
\end{gathered}
$$

Finally, $I_{6}$ can be estimated as for $I_{5}$. In fact, we note that the term $I_{5}$ involves the index $\alpha$ and $I_{6}$ involves the index $\theta$. Since $0<\theta<\frac{1}{2}<\alpha$, when we make the estimate in the case $\rho \rightarrow+\infty$ (see (3.38) $)$, the order of $\alpha$ will be dominant and hence the estimate of $I_{6}$ is much more direct. 
Now for $\alpha$ and $\gamma$ satisfying (3.3), we can obtain

$$
\begin{aligned}
& \sup _{t \in[0, T]} \int_{\mathbf{R}}\left\{\rho\left[(u-\bar{u})+\left(\varphi_{\varepsilon}^{\alpha, \theta}(\rho)\right)_{x}\right]^{2}+\rho(u-\bar{u})^{2}+\rho \Psi(\rho, \bar{\rho})\right\}(x, t) d x \\
& +\int_{0}^{T} \int_{\mathbf{R}}\left\{\bar{u}_{x}\left[p(\rho)-p(\bar{\rho})-p^{\prime}(\bar{\rho})(\rho-\bar{\rho})\right]+\rho(u-\bar{u})^{2} \bar{u}_{x}+\left(\rho^{\alpha}+\varepsilon \rho^{\theta}\right)\left[(u-\bar{u})_{x}\right]^{2}\right. \\
& \left.+\left[\left(\rho^{\frac{\alpha+\gamma-1}{2}}-\bar{\rho}^{\frac{\alpha+\gamma-1}{2}}\right)_{x}\right]^{2}+\varepsilon\left[\left(\rho^{\frac{\theta+\gamma-1}{2}}-\bar{\rho} \frac{\theta+\gamma-1}{2}\right)_{x}\right]^{2}\right\}(x, t) d x d t \\
& \leq C+C \sqrt{\varepsilon} \ln (1+T)\left\{1+\sup _{t \in[0, T]} \int_{\mathbf{R}}\left[\rho(u-\bar{u})^{2}+\rho\left(\varphi_{\varepsilon}^{\alpha, \theta}(\rho)\right)_{x}^{2}\right] d x\right\} \\
& \leq C+C \sqrt{\varepsilon} \ln (1+T)\left\{1+\sup _{t \in[0, T]} \int_{\mathbf{R}} \rho(u-\bar{u})^{2}+\rho\left[(u-\bar{u})+\left(\varphi_{\varepsilon}^{\alpha, \theta}(\rho)\right)_{x}\right]^{2} d x\right\}
\end{aligned}
$$

where $C>0$ is the constant independent of $\varepsilon$ and $t$.

Choosing $\varepsilon$ such that $\sqrt{\varepsilon} \ln (1+T) \leq \varepsilon^{\frac{1}{4}}$ and $\varepsilon$ small enough, we arrive at

$$
\begin{aligned}
& \sup _{t \in[0, T]} \int_{\mathbf{R}}\left\{\rho\left[(u-\bar{u})+\left(\varphi_{\varepsilon}^{\alpha, \theta}(\rho)\right)_{x}\right]^{2}+\rho(u-\bar{u})^{2}+\rho \Psi(\rho, \bar{\rho})\right\}(x, t) d x \\
& +\int_{0}^{T} \int_{\mathbf{R}}\left\{\bar{u}_{x}\left[p(\rho)-p(\bar{\rho})-p^{\prime}(\bar{\rho})(\rho-\bar{\rho})\right]+\rho(u-\bar{u})^{2} \bar{u}_{x}+\left(\rho^{\alpha}+\varepsilon \rho^{\theta}\right)\left[(u-\bar{u})_{x}\right]^{2}\right. \\
& \left.+\left[\left(\rho^{\frac{\alpha+\gamma-1}{2}}-\bar{\rho}^{\frac{\alpha+\gamma-1}{2}}\right)_{x}\right]^{2}+\varepsilon\left[\left(\rho^{\frac{\theta+\gamma-1}{2}}-\bar{\rho}^{\frac{\theta+\gamma-1}{2}}\right)_{x}\right]^{2}\right\}(x, t) d x d t \\
& \leq C
\end{aligned}
$$

Consequently, combining (3.29), (3.36) and (3.38) shows that for $\alpha$ and $\gamma$ satisfying (3.3), it holds that

$$
\begin{aligned}
& \sup _{t \in[0, T]} \int_{\mathbf{R}}\left\{\rho(u-\bar{u})^{2}+\left[\left(\frac{\rho^{\alpha-\frac{1}{2}}}{\alpha-\frac{1}{2}}\right)_{x}\right]^{2}+\varepsilon^{2}\left[\left(\frac{\rho^{\theta-\frac{1}{2}}}{\theta-\frac{1}{2}}\right)_{x}\right]^{2}+\rho \Psi(\rho, \bar{\rho})\right\}(x, t) d x \\
& +\int_{0}^{T} \int_{\mathbf{R}}\left\{\bar{u}_{x}\left[p(\rho)-p(\bar{\rho})-p^{\prime}(\bar{\rho})(\rho-\bar{\rho})\right]+\rho(u-\bar{u})^{2} \bar{u}_{x}+\left(\rho^{\alpha}+\varepsilon \rho^{\theta}\right)\left[(u-\bar{u})_{x}\right]^{2}\right. \\
& \left.+\left[\left(\rho^{\frac{\alpha+\gamma-1}{2}}-\bar{\rho}^{\frac{\alpha+\gamma-1}{2}}\right)_{x}\right]^{2}+\varepsilon\left[\left(\rho^{\frac{\theta+\gamma-1}{2}}-\bar{\rho}^{\frac{\theta+\gamma-1}{2}}\right)_{x}\right]^{2}\right\}(x, t) d x d t \\
& \leq C .
\end{aligned}
$$

Thus lemma 3.1 is proved.

The following lemma is the key point to get the existence of the approximate solution $\left(\rho_{\varepsilon}, u_{\varepsilon}\right)(t, x)$.

Lemma 3.2 There exist an absolutely constant $C$ and a positive constant $C(\varepsilon, T)$ depending on $\varepsilon$ and $T$ such that

$$
0<C(\varepsilon, T) \leq \rho_{\varepsilon} \leq C
$$

Proof: First, we derive the upper bound for $\rho_{\varepsilon}(x, t)$. 
It follows from the entropy estimate that

$$
\begin{aligned}
& \left(\rho_{\varepsilon}^{\alpha-\frac{1}{2}}(x, t)-\bar{\rho}^{\alpha-\frac{1}{2}}(x, t)\right)^{2}=\int_{-\infty}^{x}\left[\left(\rho_{\varepsilon}^{\alpha-\frac{1}{2}}-\bar{\rho}^{\alpha-\frac{1}{2}}\right)^{2}\right]_{x} d x \\
& =2 \int_{-\infty}^{x}\left(\rho_{\varepsilon}^{\alpha-\frac{1}{2}}-\bar{\rho}^{\alpha-\frac{1}{2}}\right)\left(\rho_{\varepsilon}^{\alpha-\frac{1}{2}}-\bar{\rho}^{\alpha-\frac{1}{2}}\right)_{x} d x \\
& \leq 2\left(\int_{-\infty}^{x}\left|\left(\rho_{\varepsilon}^{\alpha-\frac{1}{2}}-\bar{\rho}^{\alpha-\frac{1}{2}}\right)\right|^{2} d x\right)^{\frac{1}{2}}\left(\int_{-\infty}^{x}\left[\left(\rho_{\varepsilon}^{\alpha-\frac{1}{2}}-\bar{\rho}^{\alpha-\frac{1}{2}}\right)_{x}\right]^{2} d x\right)^{\frac{1}{2}} \\
& \leq C+\int_{-\infty}^{x}\left|\left(\rho_{\varepsilon}^{\alpha-\frac{1}{2}}-\bar{\rho}^{\alpha-\frac{1}{2}}\right)\right|^{2}\left[\left.\mathbf{1}\right|_{\left\{\left|\rho_{\varepsilon}-\bar{\rho}\right|<\frac{\bar{\rho}}{2}\right\}}+\left.\mathbf{1}\right|_{\left\{\left|\rho_{\varepsilon}-\bar{\rho}\right| \geq \frac{\bar{\rho}}{2}\right\}}\right] d x \\
& \equiv C+I_{1}(t)+I_{2}(t),
\end{aligned}
$$

for any fixed $t \in[0, T]$. Note that for $\left|\rho_{\varepsilon}-\bar{\rho}\right|<\frac{\bar{\rho}}{2}$, that is, $\frac{\bar{\rho}}{2}<\left|\rho_{\varepsilon}\right|<\frac{3}{2} \bar{\rho}$, one has

$$
\left|\rho_{\varepsilon}^{\alpha-\frac{1}{2}}-\bar{\rho}^{\alpha-\frac{1}{2}}\right|^{2} \leq C\left|\rho_{\varepsilon}-\bar{\rho}\right|^{2} \leq C \rho_{\varepsilon} \Psi\left(\rho_{\varepsilon}, \bar{\rho}\right) .
$$

Hence,

$$
I_{1}(t) \leq C\left\|\rho_{\varepsilon} \Psi\left(\rho_{\varepsilon}, \bar{\rho}\right)\right\|_{L^{1}(\mathbf{R})} \leq C
$$

If $\left|\rho_{\varepsilon}-\bar{\rho}\right| \geq \frac{\bar{\rho}}{2}$, then

$$
\lim _{\rho_{\varepsilon} \rightarrow 0+} \frac{\left.\left|\rho_{\varepsilon}^{\alpha-\frac{1}{2}}-\bar{\rho}^{\alpha-\frac{1}{2}}\right|^{2} \mathbf{1}\right|_{\left\{\left|\rho_{\varepsilon}-\bar{\rho}\right| \geq \frac{\bar{\rho}}{2}\right\}}}{\rho_{\varepsilon} \Psi\left(\rho_{\varepsilon}, \bar{\rho}\right)}=\bar{\rho}^{2 \alpha-1-\gamma} \leq C,
$$

and

$$
\lim _{\rho_{\varepsilon} \rightarrow+\infty} \frac{\left.\left|\rho_{\varepsilon}^{\alpha-\frac{1}{2}}-\bar{\rho}^{\alpha-\frac{1}{2}}\right|^{2} \mathbf{1}\right|_{\left\{\left|\rho_{\varepsilon}-\bar{\rho}\right| \geq \frac{\bar{\rho}}{2}\right\}}}{\rho_{\varepsilon} \Psi\left(\rho_{\varepsilon}, \bar{\rho}\right)} \leq C,
$$

if $\frac{1}{2}<\alpha \leq \frac{\gamma+1}{2}$.

Hence

$$
I_{2}(t) \leq C\left\|\rho_{\varepsilon} \Psi\left(\rho_{\varepsilon}, \bar{\rho}\right)\right\|_{L^{1}(\mathbf{R})} \leq C .
$$

It follows from (3.43), (3.44), (3.45) that

$$
\left(\rho_{\varepsilon}^{\alpha-\frac{1}{2}}-\bar{\rho}^{\alpha-\frac{1}{2}}\right)^{2} \leq C
$$

which implies that

$$
\left|\rho_{\varepsilon}\right| \leq C
$$

The upper bound of the approximate solution $\rho_{\varepsilon}(t, x)$ is proved.

Next we derive a lower bound for $\rho_{\varepsilon}(t, x)$. Since $\lim _{\rho \rightarrow 0} \rho \Psi(\rho, \bar{\rho})=\bar{\rho}^{\gamma}$, then $\rho_{\varepsilon} \Psi\left(\rho_{\varepsilon}, \bar{\rho}\right)$ is bounded away from 0 on $\left[0, \frac{1}{2} \bar{\rho}\right]$. Thus we can deduce from the bound on $\rho_{\varepsilon} \Psi\left(\rho_{\varepsilon}, \bar{\rho}\right)$ in $L^{\infty}\left(0, T ; L^{1}(\mathbf{R})\right)$ that there exists a constant $C_{1}=C_{1}(T)>0$, such that for all $t \in[0, T]$, $\operatorname{meas}\left\{x \in \mathbf{R} \mid \rho_{\varepsilon}(x, t) \leq \frac{1}{2} \bar{\rho}(x, t)\right\} \leq \frac{1}{\inf _{\rho \in\left[0, \frac{1}{2} \bar{\rho}\right]} \rho \Psi(\rho, \bar{\rho})} \int_{\left\{x \in \mathbf{R} \mid \rho_{\varepsilon}(x, t) \leq \frac{1}{2} \bar{\rho}(x, t)\right\}} \rho_{\varepsilon} \Psi\left(\rho_{\varepsilon}, \bar{\rho}\right)(x, t) d x \leq C_{1}$. 
Therefore, for every $x_{0} \in \mathbf{R}$, there exists $M=M(T)>0$ large enough, such that

$$
\begin{aligned}
\int_{\left|x-x_{0}\right| \leq M} \rho_{\varepsilon}(x, t) d x & \geq \int_{\left\{\left|x-x_{0}\right| \leq M\right\} \cap\left\{x \in \mathbf{R} \mid \rho_{\varepsilon}(x, t)>\frac{1}{2} \bar{\rho}(x, t)\right\}} \rho_{\varepsilon}(x, t) d x \\
& \geq \frac{1}{2} \inf _{(x, t)} \bar{\rho}(x, t) \operatorname{meas}\left\{\left\{\left|x-x_{0}\right| \leq M\right\} \cap\left\{x \in \mathbf{R} \mid \rho_{\varepsilon}(x, t)>\frac{1}{2} \bar{\rho}(x, t)\right\}\right\} \\
& =\frac{1}{2} \rho_{-} \operatorname{meas}\left\{\left\{\left|x-x_{0}\right| \leq M\right\} \cap\left\{x \in \mathbf{R} \mid \rho_{\varepsilon}(x, t) \leq \frac{1}{2} \bar{\rho}(x, t)\right\}^{c}\right\} \\
& \geq \frac{1}{2} \rho_{-}\left(2 M-C_{1}\right)>0
\end{aligned}
$$

for all $t \in[0, T]$.

From the continuity of $\rho_{\varepsilon}$, there exists $x_{1} \in\left[x_{0}-M, x_{0}+M\right]$ such that

$$
\rho_{\varepsilon}\left(x_{1}, t\right)=\int_{\left|x-x_{0}\right| \leq M} \rho_{\varepsilon}(x, t) d x \geq \frac{1}{2} \rho_{-}\left(2 M-C_{1}\right) .
$$

Thus,

$$
\begin{aligned}
\rho_{\varepsilon}^{\theta-\frac{1}{2}}\left(x_{0}, t\right) & =\rho_{\varepsilon}^{\theta-\frac{1}{2}}\left(x_{1}, t\right)+\int_{x_{1}}^{x_{0}}\left(\rho_{\varepsilon}^{\theta-\frac{1}{2}}\right)_{x}(x, t) d x \\
& \leq\left[\frac{1}{2} \rho_{-}\left(2 M-C_{1}\right)\right]^{\theta-\frac{1}{2}}+\left\|\left(\rho_{\varepsilon}^{\theta-\frac{1}{2}}\right)_{x}(\cdot, t)\right\|_{L^{2}(\mathbf{R})}\left|x_{1}-x_{0}\right|^{\frac{1}{2}} \\
& \leq\left[\frac{1}{2} \rho_{-}\left(2 M-C_{1}\right)\right]^{\theta-\frac{1}{2}}+C_{\varepsilon} M^{\frac{1}{2}},
\end{aligned}
$$

where we have used the fact $0<\theta<\frac{1}{2}$. Consequently, we can get that

$$
\rho_{\varepsilon}\left(x_{0}, t\right) \geq\left\{\left[\frac{1}{2} \rho_{-}\left(2 M-C_{1}\right)\right]^{\theta-\frac{1}{2}}+C_{\varepsilon} M^{\frac{1}{2}}\right\}^{\frac{2}{2 \theta-1}}:=C(\varepsilon, T) .
$$

for any $x_{0} \in \mathbf{R}$ and $t \in[0, T]$.

With the lower and upper bounds on $\rho_{\varepsilon}$, we can get the existence of the approximate solution $\left(\rho_{\varepsilon}, u_{\varepsilon}\right)(t, x)$ by a similar argument as in [26]. In order to pass the limit $\varepsilon \rightarrow 0$, we need the following higher estimates on the momentum.

Lemma 3.3 There exists a positive constant $C(T)$ independent of $\varepsilon$, such that

$$
\sup _{t \in[0, T]} \int_{\mathbf{R}} \rho_{\varepsilon}\left|u_{\varepsilon}-\bar{u}\right|^{3}(t, x) d x+\int_{0}^{T} \int_{\mathbf{R}}\left(\rho_{\varepsilon}^{\alpha}+\varepsilon \rho_{\varepsilon}^{\theta}\right)\left[\left(u_{\varepsilon}-\bar{u}\right)_{x}\right]^{2}\left|u_{\varepsilon}-\bar{u}\right| d x d t \leq C(T) .
$$

Proof: Multiplying by $(u-\bar{u})|u-\bar{u}|$ on the both sides of (3.6) yields

$$
\begin{aligned}
& \left(\frac{2}{3} \rho|u-\bar{u}|^{3}\right)_{t}+\left(\frac{2}{3} \rho u|u-\bar{u}|^{3}\right)_{x}+(p(\rho)-p(\bar{\rho}))_{x}(u-\bar{u})|u-\bar{u}| \\
& +\left[(\rho-\bar{\rho}) \bar{u}_{t}+(\rho u-\bar{\rho} \bar{u}) \bar{u}_{x}\right](u-\bar{u})|u-\bar{u}| \\
& =\left[\left(\rho^{\alpha}+\varepsilon \rho^{\theta}\right) u_{x}(u-\bar{u})|u-\bar{u}|\right]_{x}-\left(\rho^{\alpha}+\varepsilon \rho^{\theta}\right)\left[(u-\bar{u})_{x}\right]^{2}|u-\bar{u}| \\
& -\left(\rho^{\alpha}+\varepsilon \rho^{\theta}\right) \bar{u}_{x}(u-\bar{u})_{x}|u-\bar{u}|-\left(\rho^{\alpha}+\varepsilon \rho^{\theta}\right) u_{x}(u-\bar{u})(|u-\bar{u}|)_{x} .
\end{aligned}
$$

Note that

$$
(|u-\bar{u}|)_{x}=\operatorname{sgn}(u-\bar{u})(u-\bar{u})_{x}
$$


Thus

$$
\begin{aligned}
& \left(\frac{2}{3} \rho|u-\bar{u}|^{3}\right)_{t}+H_{3 x}(t, x)+2\left(\rho^{\alpha}+\varepsilon \rho^{\theta}\right)\left[(u-\bar{u})_{x}\right]^{2}|u-\bar{u}| \\
= & -(u-\bar{u})|u-\bar{u}|\left[(\rho-\bar{\rho}) \bar{u}_{t}+(\rho u-\bar{\rho} \bar{u}) \bar{u}_{x}+(p(\rho)-p(\bar{\rho}))_{x}\right] \\
& \quad-2\left(\rho^{\alpha}+\varepsilon \rho^{\theta}\right) \bar{u}_{x}(u-\bar{u})_{x}|u-\bar{u}|,
\end{aligned}
$$

where

$$
H_{3}(t, x)=\frac{2}{3} \rho u|u-\bar{u}|^{3}-\left(\rho^{\alpha}+\varepsilon \rho^{\theta}\right) u_{x}(u-\bar{u})|u-\bar{u}| .
$$

This, together with (3.17), implies

$$
\begin{aligned}
& \left(\frac{2}{3} \rho|u-\bar{u}|^{3}\right)_{t}+H_{3 x}(t, x)+2\left(\rho^{\alpha}+\varepsilon \rho^{\theta}\right)\left[(u-\bar{u})_{x}\right]^{2}|u-\bar{u}|+\rho|u-\bar{u}|^{3} \bar{u}_{x} \\
& =-p(\rho)_{x}(u-\bar{u})|u-\bar{u}|+\rho \frac{p(\bar{\rho})_{x}}{\bar{\rho}}(u-\bar{u})|u-\bar{u}|-2\left(\rho^{\alpha}+\varepsilon \rho^{\theta}\right) \bar{u}_{x}(u-\bar{u})_{x}|u-\bar{u}| \\
& :=I_{7}+I_{8}+I_{9} .
\end{aligned}
$$

Integrating (3.50) over $[0, t] \times \mathbf{R}$ with respect to $t, x$ gives

$$
\begin{aligned}
& \int_{\mathbf{R}} \frac{2}{3} \rho|u-\bar{u}|^{3}(t, x) d x+\int_{0}^{t} \int_{\mathbf{R}}\left[2\left(\rho^{\alpha}+\varepsilon \rho^{\theta}\right)\left[(u-\bar{u})_{x}\right]^{2}|u-\bar{u}|^{\delta}+\rho|u-\bar{u}|^{3} \bar{u}_{x}\right] d x d \tau \\
& =\int_{0}^{t} \int_{\mathbf{R}}\left(I_{7}+I_{8}+I_{9}\right) d x d \tau .
\end{aligned}
$$

Now we estimate such term on the right hand side of (3.51). First, integrating by parts with respect to $x$ gives

$$
\begin{aligned}
& \int_{0}^{t} \int_{\mathbf{R}} I_{7} d x d \tau=2 \int_{0}^{t} \int_{\mathbf{R}} p(\rho)(u-\bar{u})_{x}|u-\bar{u}| d x d \tau \\
& \leq \int_{0}^{t} \int_{\mathbf{R}} \rho^{\alpha}\left[(u-\bar{u})_{x}\right]^{2} d x d \tau+\int_{0}^{t} \int_{\mathbf{R}} \rho^{2 \gamma-\alpha-1} \rho|u-\bar{u}|^{2} d x d \tau \\
& \leq C
\end{aligned}
$$

uniformly with respect to $\varepsilon$ if

$$
2 \gamma-\alpha-1 \geq 0, \quad \text { i. e. } \quad \alpha \leq 2 \gamma-1
$$

In fact, the relation (3.4) guarantees (3.53) because $\frac{\gamma+1}{2}<2 \gamma-1$.

Next,

$$
\begin{aligned}
\int_{0}^{t} \int_{\mathbf{R}} I_{8} d x d \tau & =\int_{0}^{t} \int_{\mathbf{R}} \rho \frac{p(\bar{\rho})_{x}}{\bar{\rho}}(u-\bar{u})|u-\bar{u}| d x d \tau \\
& \leq C \sup _{t \in[0, T]} \int_{\mathbf{R}} \rho|u-\bar{u}|^{2}(t, x) d x \int_{0}^{T}\left\|\bar{\rho}_{x}\right\|_{L^{\infty}(\mathbf{R})} d t \\
& \leq C \sup _{t \in[0, T]} \int_{\mathbf{R}} \rho|u-\bar{u}|^{2}(t, x) d x \int_{0}^{T}(1+t)^{-1} d t \\
& \leq C_{1} \ln (1+T) .
\end{aligned}
$$


Finally,

$$
\begin{aligned}
\int_{0}^{t} \int_{\mathbf{R}} I_{9} d x d \tau & =\int_{0}^{t} \int_{\mathbf{R}}-2\left(\rho^{\alpha}+\varepsilon \rho^{\theta}\right) \bar{u}_{x}(u-\bar{u})_{x}|u-\bar{u}| d x d \tau \\
& \leq \int_{0}^{t} \int_{\mathbf{R}}\left(\rho^{\alpha}+\varepsilon \rho^{\theta}\right)\left[(u-\bar{u})_{x}\right]^{2}|u-\bar{u}| d x d \tau+\int_{0}^{t} \int_{\mathbf{R}}\left(\rho^{\alpha}+\varepsilon \rho^{\theta}\right) \bar{u}_{x}^{2}|u-\bar{u}| d x d \tau
\end{aligned}
$$

One also has

$$
\begin{aligned}
& \int_{0}^{t} \int_{\mathbf{R}}\left(\rho^{\alpha}+\varepsilon \rho^{\theta}\right) \bar{u}_{x}^{2}|u-\bar{u}| d x d \tau \\
& \leq \int_{0}^{t} \int_{\mathbf{R}} \rho|u-\bar{u}|^{3} d x d \tau+C \int_{0}^{t} \int_{\mathbf{R}}\left(\rho^{\frac{3 \alpha-1}{2}}+\varepsilon^{\frac{3}{2}} \rho^{\frac{3 \theta-1}{2}}\right) \bar{u}_{x}^{3} d x d \tau \\
& \leq \int_{0}^{t} \int_{\mathbf{R}}^{t} \rho|u-\bar{u}|^{3} d x d \tau+C \int_{0}^{t}\left\|\bar{u}_{x}\right\|_{L^{3}(\mathbf{R})}^{3} d \tau \\
& \leq \int_{0}^{t} \int_{\mathbf{R}}^{t} \rho|u-\bar{u}|^{3} d x d \tau+C \int_{0}^{t}(1+\tau)^{-2} d \tau \\
& \leq \int_{0}^{t} \int_{\mathbf{R}} \rho|u-\bar{u}|^{3} d x d \tau+C
\end{aligned}
$$

if $\alpha, \theta \geq \frac{1}{3}$. Without loss of generality, we can set $\theta=\frac{1}{3}$.

Substituting (3.52)-(3.56) into (3.51) implies Lemma 3.3.

Now with these uniform in $\varepsilon$ estimates in hand, we can pass the limit process $\varepsilon \rightarrow 0$, obtain the existence of the weak solution $(\rho, u)(t, x)$, and get the uniform in time estimates in Theorem 2.1 .

\section{Asymptotic behavior of weak solutions}

In this section, we will study the asymptotic behavior of the weak solution $(\rho, u)(t, x)$ obtained in previous section. We assume that the solution is smooth enough. The rigorous proof can be obtained by using the usual regularization procedure.

Proof of Theorem 2.2. Since $0 \leq \rho \leq C, 0<\rho_{-}<\bar{\rho}<\rho_{+}$, it holds that

$$
C_{1}^{-1}(\rho-\bar{\rho})^{2} \leq \rho \Psi(\rho, \bar{\rho}) \leq C_{1}(\rho-\bar{\rho})^{2}
$$

for some constant $C_{1}>0$ which may depend on $C, \rho_{-}, \rho_{+}$.

In the following, we denote by $C>0$ a universal constant. For any $s \geq 1$, Lemma 3.2 implies

$$
\left|\rho^{s}-\bar{\rho}^{s}\right|^{2} \leq C|\rho-\bar{\rho}|^{2} .
$$

Hence

$$
\int_{\mathbf{R}}\left|\rho^{s}-\bar{\rho}^{s}\right|^{2} d x \leq C \int_{\mathbf{R}}|\rho-\bar{\rho}|^{2} d x \leq C
$$

Similarly,

$$
\int_{\mathbf{R}}\left|\rho^{s}-\bar{\rho}^{s}\right|^{2 \lambda} d x \leq C \int_{\mathbf{R}}|\rho-\bar{\rho}|^{2 \lambda} d x \leq C
$$


for any $\lambda \geq 1$. Moreover, one has

$$
\begin{aligned}
& \int_{\mathbf{R}}\left|\left[\left(\rho^{s}-\bar{\rho}^{s}\right)^{2 \lambda}\right]_{x}\right| d x=2 \lambda s \int_{\mathbf{R}}\left|\left(\rho^{s}-\bar{\rho}^{s}\right)^{2 \lambda-1}\left[\rho^{s-1} \rho_{x}-\bar{\rho}^{s-1} \bar{\rho}_{x}\right]\right| d x \\
& \leq \frac{2 \lambda s(2 \alpha-1)}{2}\left(\int_{\mathbf{R}}\left(\rho^{s}-\bar{\rho}^{s}\right)^{2(2 \lambda-1)} \rho^{2 s+1-2 \alpha} d x\right)^{\frac{1}{2}}\left(\int_{\mathbf{R}}\left[\left(\rho^{\alpha-\frac{1}{2}}\right)_{x}\right]^{2} d x\right)^{\frac{1}{2}} \\
& \quad+2 \lambda s\left(\int_{\mathbf{R}}\left|\rho^{s}-\bar{\rho}^{s}\right|^{2(2 \lambda-1)} d x\right)^{\frac{1}{2}}\left(\int_{\mathbf{R}} \bar{\rho}^{2(s-1)}\left|\bar{\rho}_{x}\right|^{2} d x\right)^{\frac{1}{2}} \\
& \leq C .
\end{aligned}
$$

It follows from (4.3) that for any fixed $t$,

$$
\rho^{s}-\bar{\rho}^{s} \rightarrow 0
$$

as $|x| \rightarrow \infty$. By Lemma 3.1, it holds that

$$
\int_{0}^{t} \int_{\mathbf{R}}\left[\left(\rho^{\frac{\gamma+\alpha-1}{2}}-\bar{\rho}^{\frac{\gamma+\alpha-1}{2}}\right)_{x}\right]^{2} d x d t \leq C,
$$

with $C$ an absolute constant depending only on the initial data. Set $b=\frac{\gamma+\alpha-1}{2}$. Then

$$
\int_{0}^{t} \int_{\mathbf{R}}\left[\left(\rho^{b}-\bar{\rho}^{b}\right)_{x}\right]^{2} d x d t \leq C .
$$

Choosing $s>b+1$, one has

$$
\begin{aligned}
& \left(\rho^{s}-\bar{\rho}^{s}\right)^{2}(t, x)=\int_{-\infty}^{x}\left[\left(\rho^{s}-\bar{\rho}^{s}\right)^{2}\right]_{x} d x=2 \int_{-\infty}^{x}\left(\rho^{s}-\bar{\rho}^{s}\right)\left(\rho^{s}-\bar{\rho}^{s}\right)_{x} d x \\
& =2 s \int_{-\infty}^{x}\left(\rho^{s}-\bar{\rho}^{s}\right)\left(\rho^{s-1} \rho_{x}-\bar{\rho}^{s-1} \bar{\rho}_{x}\right) d x \\
& =\frac{2 s}{b} \int_{-\infty}^{x}\left(\rho^{s}-\bar{\rho}^{s}\right)\left[\left(\rho^{b}-\bar{\rho}^{b}\right)_{x} \rho^{s-b}+\left(\bar{\rho}^{b}\right)_{x}\left(\rho^{s-b}-\bar{\rho}^{s-b}\right)\right] d x \\
& \leq C\left\|\rho^{s}-\bar{\rho}^{s}\right\|_{L^{2}(\mathbf{R})}\left\|\left(\rho^{b}-\bar{\rho}^{b}\right)_{x}\right\|_{L^{2}(\mathbf{R})}+C \int_{\mathbf{R}}\left(\bar{\rho}^{b}\right)_{x}\left(\rho^{s}-\bar{\rho}^{s}\right)\left(\rho^{s-b}-\bar{\rho}^{s-b}\right) d x \\
& \leq\left\|\rho^{s}-\bar{\rho}^{s}\right\|_{L^{2}(\mathbf{R})}\left\|\left(\rho^{b}-\bar{\rho}^{b}\right)_{x}\right\|_{L^{2}(\mathbf{R})}+C \int_{\mathbf{R}} \bar{u}_{x}\left[\rho^{\gamma}-\bar{\rho}^{\gamma}-\gamma \bar{\rho}^{\gamma-1}(\rho-\bar{\rho})\right] d x
\end{aligned}
$$

where in the last inequality, we have used the fact that

$$
\begin{aligned}
& \left(\bar{\rho}^{b}\right)_{x}\left(\rho^{s}-\bar{\rho}^{s}\right)\left(\rho^{s-b}-\bar{\rho}^{s-b}\right) \\
& =b \bar{\rho}^{b-1} \bar{\rho}_{x}\left(\rho^{s}-\bar{\rho}^{s}\right)\left(\rho^{s-b}-\bar{\rho}^{s-b}\right) \\
& =\frac{b \bar{\rho}^{b}}{\sqrt{p^{\prime}(\bar{\rho})}} \bar{u}_{x}\left(\rho^{s}-\bar{\rho}^{s}\right)\left(\rho^{s-b}-\bar{\rho}^{s-b}\right) \\
& \leq C \bar{u}_{x}\left[\rho^{\gamma}-\bar{\rho}^{\gamma}-\gamma \bar{\rho}^{\gamma-1}(\rho-\bar{\rho})\right] .
\end{aligned}
$$


Consequently,

$$
\begin{aligned}
& \int_{0}^{t} \sup _{x \in \mathbf{R}}\left(\rho^{s}-\bar{\rho}^{s}\right)^{4} d t \leq C \sup _{t \in[0, T]}\left\|\rho^{s}-\bar{\rho}^{s}\right\|_{L^{2}(\mathbf{R})}^{2} \int_{0}^{t}\left\|\left(\rho^{b}-\bar{\rho}^{b}\right)_{x}\right\|_{L^{2}(\mathbf{R})}^{2} d t \\
& \quad+C \sup _{t \in[, T]} \int_{\mathbf{R}}\left[\rho^{\gamma}-\bar{\rho}^{\gamma}-\gamma \bar{\rho}^{\gamma-1}(\rho-\bar{\rho})\right] d x \int_{0}^{t} \int_{\mathbf{R}} \bar{u}_{x}\left[\rho^{\gamma}-\bar{\rho}^{\gamma}-\gamma \bar{\rho}^{\gamma-1}(\rho-\bar{\rho})\right] d x d \tau \\
& \leq C .
\end{aligned}
$$

Moreover, applying (4.3) leads to

$$
\begin{aligned}
& \int_{0}^{t} \int_{\mathbf{R}}\left(\rho^{s}-\bar{\rho}^{s}\right)^{4}\left(\rho^{s}-\bar{\rho}^{s}\right)^{2 l} d x d t \\
& \leq \int_{0}^{t}\left[\sup _{x \in \mathbf{R}}\left(\rho^{s}-\bar{\rho}^{s}\right)^{4} \int_{\mathbf{R}}\left(\rho^{s}-\bar{\rho}^{s}\right)^{2 l} d x\right] d t \\
& \leq \sup _{t} \int_{\mathbf{R}}\left(\rho^{s}-\bar{\rho}^{s}\right)^{2 l} d x \int_{0}^{t} \sup _{x \in \mathbf{R}}\left(\rho^{s}-\bar{\rho}^{s}\right)^{4} d t \leq C,
\end{aligned}
$$

where $l \geq 1$ is any real number. Hence

$$
\int_{0}^{t} \int_{\mathbf{R}}\left(\rho^{s}-\bar{\rho}^{s}\right)^{4+2 l} d x d t \leq C
$$

Denote $f(t)=\int_{\mathbf{R}}\left(\rho^{s}-\bar{\rho}^{s}\right)^{4+2 l} d x$. Then $f(t) \in L^{1}(0, \infty) \cap L^{\infty}(0, \infty)$ due to (4.3) and (4.12). Furthermore, direct calculations show that

$$
\begin{aligned}
\frac{d}{d t} f(t)= & (4+2 l) s \int_{\mathbf{R}}\left(\rho^{s}-\bar{\rho}^{s}\right)^{3+2 l}\left(\rho^{s-1} \rho_{t}-\bar{\rho}^{s-1} \bar{\rho}_{x}\right) d x \\
= & -(4+2 l) s \int_{\mathbf{R}}\left(\rho^{s}-\bar{\rho}^{s}\right)^{3+2 l}\left[\rho^{s-1}(\rho u)_{x}-\bar{\rho}^{s-1}(\bar{\rho} \bar{u})_{x}\right] d x \\
= & (4+2 l)(3+2 l) s \int_{\mathbf{R}}\left(\rho^{s}-\bar{\rho}^{s}\right)^{2+2 l}\left(\rho^{s}-\bar{\rho}^{s}\right)_{x}\left(\rho^{s-1} \rho u-\bar{\rho}^{s-1} \bar{\rho} \bar{u}\right) d x \\
& +(4+2 l) s(s-1) \int_{\mathbf{R}}\left(\rho^{s}-\bar{\rho}^{s}\right)^{3+2 l}\left(\rho^{s-2} \rho_{x} \rho u-\bar{\rho}^{s-2} \bar{\rho}_{x} \bar{\rho} \bar{u}\right) d x \\
= & (4+2 l)(3+2 l) s \int_{\mathbf{R}}\left(\rho^{s}-\bar{\rho}^{s}\right)^{2+2 l}\left(\rho^{s}-\bar{\rho}^{s}\right)_{x}\left(\rho^{s} u-\bar{\rho}^{s} \bar{u}\right) d x \\
& +(4+2 l) s(s-1) \int_{\mathbf{R}}\left(\rho^{s}-\bar{\rho}^{s}\right)^{3+2 l}\left(\rho^{s-1} \rho_{x} u-\bar{\rho}^{s-1} \bar{\rho}_{x} \bar{u}\right) d x \\
= & (4+2 l)(3+2 l) s \int_{\mathbf{R}}\left(\rho^{s}-\bar{\rho}^{s}\right)^{2+2 l}\left(\rho^{s}-\bar{\rho}^{s}\right)_{x} \rho^{s}(u-\bar{u}) d x \\
& +(4+2 l)(3+2 l) s \int_{\mathbf{R}}\left(\rho^{s}-\bar{\rho}^{s}\right)^{2+2 l}\left(\rho^{s}-\bar{\rho}^{s}\right)_{x} \bar{u}\left(\rho^{s}-\bar{\rho}^{s}\right) d x \\
& +(4+2 l) s(s-1) \int_{\mathbf{R}}\left(\rho^{s}-\bar{\rho}^{s}\right)^{3+2 l} \rho^{s-1} \rho_{x}(u-\bar{u}) d x \\
& +(4+2 l) s(s-1) \int_{\mathbf{R}}\left(\rho^{s}-\bar{\rho}^{s}\right)^{3+2 l} \bar{u}\left(\rho^{s-1} \rho_{x}-\bar{\rho}^{s-1} \bar{\rho}_{x}\right) d x \\
:= & J_{1}(t)+J_{2}(t)+J_{3}(t)+J_{4}(t) .
\end{aligned}
$$


Now we claim that $J_{i}(t) \in L^{2}(0,+\infty),(i=1,2,3,4)$. In fact,

$$
\begin{aligned}
J_{1}(t)= & \frac{(4+2 l)(3+2 l) s^{2}}{b} \int_{\mathbf{R}}\left(\rho^{s}-\bar{\rho}^{s}\right)^{2+2 l} \rho^{s}(u-\bar{u})\left[\rho^{s-b}\left(\rho^{b}-\bar{\rho}^{b}\right)_{x}+\left(\rho^{s-b}-\bar{\rho}^{s-b}\right)\left(\bar{\rho}^{b}\right)_{x}\right] d x \\
= & \frac{(4+2 l)(3+2 l) s^{2}}{b} \int_{\mathbf{R}}\left(\rho^{s}-\bar{\rho}^{s}\right)^{2+2 l} \sqrt{\rho}(u-\bar{u}) \rho^{2 s-b-\frac{1}{2}}\left(\rho^{b}-\bar{\rho}^{b}\right)_{x} d x \\
& +\frac{(4+2 l)(3+2 l) s^{2}}{b} \int_{\mathbf{R}}\left(\rho^{s}-\bar{\rho}^{s}\right)^{2+2 l} \sqrt{\rho}(u-\bar{u}) \rho^{s-\frac{1}{2}}\left(\rho^{s-b}-\bar{\rho}^{s-b}\right)\left(\bar{\rho}^{b}\right)_{x} d x \\
\leq & C\|\sqrt{\rho}(u-\bar{u})\|_{L^{2}(\mathbf{R})}\left\|\left(\rho^{b}-\bar{\rho}^{b}\right)_{x}\right\|_{L^{2}(\mathbf{R})} \\
& +C\|\sqrt{\rho}(u-\bar{u})\|_{L^{2}(\mathbf{R})}\left\|\left(\rho^{s}-\bar{\rho}^{s}\right)^{2+2 l}\left(\rho^{s-b}-\bar{\rho}^{s-b}\right)\left(\bar{\rho}^{b}\right)_{x}\right\|_{L^{2}(\mathbf{R})} \\
\leq & C\|\sqrt{\rho}(u-\bar{u})\|_{L^{2}(\mathbf{R})}\left\|\left(\rho^{b}-\bar{\rho}^{b}\right)_{x}\right\|_{L^{2}(\mathbf{R})} \\
& +C\|\sqrt{\rho}(u-\bar{u})\|_{L^{2}(\mathbf{R})}\left\|\bar{u}_{x}\left[\rho^{\gamma}-\bar{\rho}^{\gamma}-\gamma \bar{\rho}^{\gamma-1}(\rho-\bar{\rho})\right]\right\|_{L^{2}(\mathbf{R})}
\end{aligned}
$$

Thus,

$$
\begin{aligned}
\int_{0}^{t}\left|J_{1}(t)\right|^{2} d t \leq & C \sup _{t \in[0, T]}\|\sqrt{\rho}(u-\bar{u})\|_{L^{2}(\mathbf{R})}^{2} \int_{0}^{t}\left\|\left(\rho^{b}-\bar{\rho}^{b}\right)_{x}\right\|_{L^{2}(\mathbf{R})}^{2} d t \\
& +C \sup _{t \in[0, T]}\|\sqrt{\rho}(u-\bar{u})\|_{L^{2}(\mathbf{R})}^{2} \int_{0}^{t}\left\|\bar{u}_{x}\left[\rho^{\rho}-\bar{\rho}^{\gamma}-\gamma \bar{\rho}^{\gamma-1}(\rho-\bar{\rho})\right]\right\|_{L^{2}(\mathbf{R})}^{2} d t \\
\leq & C \sup _{t \in[0, T]}\|\sqrt{\rho}(u-\bar{u})\|_{L^{2}(\mathbf{R})}^{2} \int_{0}^{t}\left\|\left(\rho^{b}-\bar{\rho}^{b}\right)_{x}\right\|_{L^{2}(\mathbf{R})}^{2} d t \\
& +C \sup _{t \in[0, T]}\|\sqrt{\rho}(u-\bar{u})\|_{L^{2}(\mathbf{R})}^{2} \sup _{t \in[0, T]}\|\rho \Psi(\rho, \bar{\rho})\|_{L^{1}(\mathbf{R})} \cdot \\
& \int_{0}^{t} \int_{\mathbf{R}} \bar{u}_{x}\left[\rho^{\gamma}-\bar{\rho}^{\gamma}-\gamma \bar{\rho}^{\gamma-1}(\rho-\bar{\rho})\right] d x d t \\
\leq & C .
\end{aligned}
$$

The fact that $J_{i}(t) \in L^{2}(0,+\infty),(i=2,3,4)$ can be shown similarly.

Hence

$$
\frac{d}{d t} f(t) \in L^{2}(0,+\infty)
$$

Combining the obtained fact that $f(t) \in L^{1}(0,+\infty) \cap L^{\infty}(0,+\infty)$, one has

$$
f(t) \rightarrow 0, \quad t \rightarrow+\infty .
$$

Letting $m \geq 1$ be any real number to be determined later, we have

$$
\begin{aligned}
\left|\left(\rho^{s}-\bar{\rho}^{s}\right)^{m}\right| & =\left|\int_{-\infty}^{x}\left[\left(\rho^{s}-\bar{\rho}^{s}\right)^{m}\right]_{x} d x\right| \\
& =\left|m \int_{-\infty}^{x}\left(\rho^{s}-\bar{\rho}^{s}\right)^{m-1}\left(\rho^{s}-\bar{\rho}^{s}\right)_{x} d x\right| \\
& =\left|m \int_{-\infty}^{x}\left(\rho^{s}-\bar{\rho}^{s}\right)^{m-1}\left[\frac{s}{\alpha-\frac{1}{2}}\left(\rho^{\alpha-\frac{1}{2}}\right)_{x} \rho^{s-\alpha+\frac{1}{2}}-s \bar{\rho}^{s-1} \bar{\rho}_{x}\right] d x\right| \\
& \leq C\left\|\left(\rho^{s}-\bar{\rho}^{s}\right)^{m-1}\right\|_{L^{2}(\mathbf{R})}\left[\left\|\left(\rho^{\alpha-\frac{1}{2}}\right)_{x}\right\|_{L^{2}(\mathbf{R})}+\left\|\bar{\rho}_{x}\right\|_{L^{2}(\mathbf{R})}\right] \\
& \leq C\left\|\left(\rho^{s}-\bar{\rho}^{s}\right)^{m-1}\right\|_{L^{2}(\mathbf{R})}
\end{aligned}
$$


Choose $2(m-1)=4+2 l$ to get

$$
\sup _{x \in \mathbf{R}}\left|\left(\rho^{s}-\bar{\rho}^{s}\right)^{m}\right| \leq C f^{\frac{1}{2}}(t) \rightarrow 0
$$

as $t \rightarrow+\infty$.

Therefore,

$$
\lim _{t \rightarrow+\infty} \sup _{x \in \mathbf{R}}\left|\rho^{s}-\bar{\rho}^{s}\right|=0 .
$$

Now we prove that $\lim _{t \rightarrow+\infty} \sup _{x \in \mathbf{R}}|\rho-\bar{\rho}|=0$. Using the fact that

$$
\begin{aligned}
|\rho-\bar{\rho}|^{s} & =|\rho-\bar{\rho}|^{s} \mathbf{1}_{\left\{0 \leq \rho \leq \frac{\rho_{-}}{2}\right\}}+|\rho-\bar{\rho}|^{s} \mathbf{1}_{\left\{\rho>\frac{\rho_{-}}{2}\right\}} \\
& \leq C\left|\rho^{s}-\bar{\rho}^{s}\right| \mathbf{1}_{\left\{0 \leq \rho \leq \frac{\rho_{-}}{2}\right\}}+C\left|\rho^{s}-\bar{\rho}^{s}\right|^{s} \mathbf{1}_{\left\{\rho>\frac{\rho_{-}}{2}\right\}} .
\end{aligned}
$$

Therefore, we have

$$
\sup _{x \in \mathbf{R}}|\rho-\bar{\rho}|^{s} \leq C \sup _{x \in \mathbf{R}}\left|\rho^{s}-\bar{\rho}^{s}\right|+C \sup _{x \in \mathbf{R}}\left|\rho^{s}-\bar{\rho}^{s}\right|^{s} \rightarrow 0,
$$

as $t \rightarrow+\infty$, which implies that

$$
\lim _{t \rightarrow \infty} \sup _{x \in \mathbf{R}}|\rho-\bar{\rho}|=0
$$

The proof of the lemma is finished.

\section{Vanishing of vacuum states and blow-up phenom- ena}

In this subsection, we first give a sketch of proof of Theorem 2.3 and then give some remarks on the blow-up phenomena of the solutions when the vacuum states vanish. These results are similar to those in [18] in which the initial-boundary value problem and periodic boundary value problem are studied.

Proof: [Sketch of proof of Theorem 2.3]

It follows from Theorem 2.2 that for any $0<\rho_{1}<\inf _{t, x} \bar{\rho}(t, x)$, there exists a time $T_{0}>0$ such that

$$
0<\rho_{1} \leq \rho(x, t) \leq C, \quad(x, t) \in \mathbf{R} \times\left[T_{0}, \infty\right) .
$$

Therefore, for $t \geq T_{0}$, the density is bounded away from the zero and the vacuum states vanish. Using the estimate in (2.14) and the standard theory for linear parabolic equations, one can obtain that for $t \geq T_{0}$, the weak solution becomes a strong solution to (1.1)-(1.2), satisfying

$$
\left\{\begin{array}{l}
\rho-\bar{\rho} \in L^{\infty}\left(T_{0}, t ; H^{1}(\mathbf{R})\right), \quad \rho_{t} \in L^{\infty}\left(T_{0}, t ; L^{2}(\mathbf{R})\right) \\
u-\bar{u} \in L^{2}\left(T_{0}, t ; H^{2}(\mathbf{R})\right), \quad u_{t} \in L^{2}\left(T_{0}, t ; L^{2}(\mathbf{R})\right)
\end{array}\right.
$$


The detail of the proof is referred to [18] and is omitted it here. Furthermore, the asymptotic behaviors $\lim _{t \rightarrow \infty} \sup _{x \in \mathbf{R}}|\rho-\bar{\rho}|=0$ and $\lim _{t \rightarrow \infty}\|\rho-\bar{\rho}\|_{L^{p}}=0$ for $2<p \leq \infty$ follow directly from (2.16) and the estimate $\|\rho-\bar{\rho}\|_{L^{2}} \leq C$. The asymptotic behavior on the velocity $\lim _{t \rightarrow \infty} \| u-$ $\bar{u} \|_{L^{2}}=0$ follows from the standard arguments, see [28] for instance.

It should be remarked that we also have finite blow-up phenomena for the weak solutions of the Cauchy problem (1.1)-(1.3) at the time when the vacuum states vanish if the density contains vacuum states at least at one point. These are similar as in [18] in which the 1D initial-boundary value problem and periodic problem are investigated. To be more precise, we note that, if the density contains vacuum states at least at one point, due to the facts that $\rho \in C(\mathbf{R} \times[0, T])$ for any $T>0$ and $\lim _{t \rightarrow \infty} \sup _{x \in \mathbf{R}}|\rho-\bar{\rho}|=0$, there exists some critical time $T_{1} \in\left[0, T_{0}\right)$ with $T_{0}>0$ given by (5.1) and a nonempty subset $\Omega^{0} \subset \mathbf{R}$ such that

$$
\left\{\begin{array}{l}
\rho\left(x, T_{1}\right)=0, \forall x \in \Omega^{0}, \\
\rho\left(x, T_{1}\right)>0, \forall x \in \mathbf{R} \backslash \Omega^{0}, \\
\rho(x, t)>0, \forall(x, t) \in \mathbf{R} \times\left(T_{1}, T_{0}\right] .
\end{array}\right.
$$

From (5.2), it is easy to know that for any $\delta>0$,

$$
\int_{T_{1}+\delta}^{T_{0}}\left\|u_{x}\right\|_{L^{\infty}} d s \leq \int_{T_{1}+\delta}^{T_{0}}\left\|(u-\bar{u})_{x}\right\|_{L^{\infty}} d s+\int_{T_{1}+\delta}^{T_{0}}\left\|\bar{u}_{x}\right\|_{L^{\infty}} d s<\infty .
$$

However, one has the following blow-up result of the solution.

Theorem 5.1 Let $(\rho, u)$ be any global weak solution, which contains vacuum states at least at one point for some time, to the Cauchy problem (1.1)-(1.3) satisfying (2.13)-(2.14). Let $T_{0}>0$ and $T_{1} \in\left[0, T_{0}\right)$ be the time such that (5.1) and (5.3) holds respectively. Then, the solution $(\rho, u)$ blows up as vacuum states vanish in the following sense: for any $\eta>0$, it holds

$$
\lim _{t \rightarrow T_{1}^{+}} \int_{t}^{T_{1}+\eta}\left\|u_{x}\right\|_{L^{\infty}} d s=\infty
$$

On the other hand, if there exists some $T_{2} \in\left(0, T_{0}\right)$ such that the weak solution $(\rho, u)$ satisfies

$$
\|u-\bar{u}\|_{L^{1}\left(0, T_{2} ; W^{1, \infty}(\mathbf{R})\right)}<\infty,
$$

then, there is a time $T_{3} \in\left[T_{2}, T_{0}\right)$ such that

$$
\lim _{t \rightarrow T_{3}^{-}} \int_{0}^{t}\left\|u_{x}\right\|_{L^{\infty}} d s=\infty .
$$

The proof of Theorem 5.1 is completely similar to that in [18]. For completeness, we just sketch it here.

Proof It suffices to prove (5.4) since the proof of (5.5) is similar. If (5.4) is not true, then there exists a fixed constant $\eta>0$, such that

$$
\int_{T_{1}}^{T_{1}+\eta}\left\|u_{x}\right\|_{L^{\infty}} d s<\infty
$$


Thanks to (5.2) and (5.6), the particle path $x(s)=X(s ; t, x)$ through $(x, t) \in R \times\left(T_{1}, T_{1}+\eta\right]$ can be well defined by solving

$$
\begin{cases}\frac{\partial}{\partial s} X(s ; t, x)=u(X(s ; t, x), s), & T_{1} \leq s<T_{1}+\eta, \\ X(t ; t, x)=x, & T_{1} \leq t<T_{1}+\eta, x \in R .\end{cases}
$$

Then by the continuity equation (1.1), one has

$$
\rho(x, t)=\rho\left(X\left(T_{1} ; t, x\right), T_{1}\right) \exp \left\{-\left.\int_{T_{1}}^{t} u_{y}(y, s)\right|_{y=X(s ; t, x)} d s\right\}
$$

for any $(x, t) \in R \times\left(T_{1}, T_{1}+\eta\right]$. It follows from (5.6) and (5.7) that for $x_{1} \in \Omega_{0}$ defined by (5.3), which satisfies $\rho\left(x_{1}, T_{1}\right)=0$, there exists a trajectory $x=x_{1}(t) \in R$ for $t \in\left[T_{1}, T_{1}+\eta\right]$ so that $X\left(T_{1} ; t, x_{1}(t)\right)=x_{1}$. Thus, due to (5.8) and (5.6), one has that $\rho\left(x_{1}(t), t\right)=0$ for all $t \in\left(T_{1}, T_{1}+\eta\right]$, which is a contradiction to (5.3). (5.4) is then proved and the proof of the theorem is finished.

\section{References}

[1] D. Bresch and B. Desjardins, Existence of global weak solutions for a 2D viscous shallow water equations and convergence to the quasi-geostrophic model, Comm. Math. Phys., 238(1-2) (2003) 211-223.

[2] D. Bresch, B. Desjardins, Chi-Kun Lin, On some compressible fluid models: Korteweg, lubrication, and shallow water systems, Comm. Partial Differential Equations, 28(3-4)( 2003) 843-868.

[3] D. Bresch and B. Desjardins, Some diffusive capillary models of Korteweg type, $C . R$. Math. Acad. Sci. Paris, Section Mécanique, 332(2004), No. 11, 881-886.

[4] D. Bresch and B. Desjardins, On the construction of approximate solutions for the 2D viscous shallow water model and for compressible Navier-Stokes models, J. Math. Pures Appl. 86 (2006) 362-368.

[5] D. Bresch, B. Desjardins, D. Gerard-Varet, On compressible Navier-Stokes equations with density dependent viscosities in bounded domains, J. Math. Pures Appl., 87(2) (2007) $227-235$.

[6] D. Y. Fang, T. Zhang, Compressible Navier-Stokes equations with vacuum state in the case of general pressure law, Math. Methods Appl. Sci., 29 (2006), 1081-1106.

[7] E. Feireisl, A. Novotný and H. Petzeltová, On the existence of globally defined weak solutions to the Navier-Stokes equations of isentropic compressible fluids, J. Math. Fluid Mech. 3 (2001) 358-392.

[8] J. F. Gerbeau, B. Perthame, Derivation of viscous Saint-Venant system for laminar shallow water; numerical validation, Discrete Contin. Dyn. Syst. Ser. B 1 (2001), 89-102. 
[9] Z. H. Guo, Q. S. Jiu, Z. P. Xin, Spherically symmetric isentropic compressible flows with density-dependent viscosity coefficients, SIAM J. Math. Anal. 39 (2008), No.5, 1402-1427.

[10] D. Hoff, Global existence for 1D, compressible, isentropic Navier-Stokes equations with large initial data, Trans. Amer. Math. Soc., 303(1)(1987), 169-181.

[11] D. Hoff, Strong convergence to global solutions for multidimensional flows of compressible, viscous fluids with polytropic equations of state and discontinuous initial data, Arch. Rat. Mech. Anal., 132(1995), 1-14.

[12] D. Hoff, D. Serre, The failure of continuous dependence on initial data for the NavierStokes equations of compressible flow, SIAM J. Appl. Math. 51(1991) 887-898.

[13] D. Hoff, J. Smoller, Non-formation of vacuum states for compressible Navier- Stokes equations. Comm. Math. Phys. 216 (2001), no. 2, 255-276.

[14] S. Jiang, Global smooth solutions of the equations of a viscous, heat-conducting onedimensional gas with density-dependent viscosity, Math. Nachr. 190(1998) 169-183.

[15] S. Jiang, Z. P. Xin and P. Zhang, Global weak solutions to 1D compressible isentropy Navier-Stokes with density-dependent viscosity, Methods and Applications of Analysis 12 (3)(2005) 239-252.

[16] Q. S. Jiu, Z.P. Xin, The Cauchy problem for 1D compressible flows with density-dependent viscosity coefficients. Kinet. Relat. Models 1 (2), (2008), 313-330.

[17] A. V. Kazhikhov, V. V. Shelukhin, Unique global solution with respect to time of initialboundary value problems for one-dimensional equations of a viscous gas, J. Appl. Math. Mech. 41(1977) 273-282; translated from Prikl. Mat. Meh. 41 (1977) 282-291.

[18] H. L., Li, J. Li, Z. P. Xin, Vanishing of Vacuum States and Blow-up Phenomena of the Compressible Navier-Stokes Equations, Comm. Math. Phys., 281(2), (2008), 401-444.

[19] P. L. Lions, Mathematical Topics in Fluid Dynamics 2, Compressible Models, Oxford Science Publication, Oxford, 1998.

[20] T. Liu, Z. Xin, Nonlinear stability of rarefaction waves for compressible Navier-Stokes equations, Comm. Math. Phys., 118 (1988), 451-465.

[21] T. P. Liu, Z. P. Xin, T. Yang, Vacuum states of compressible flow, Discrete Continuous Dynam. systems4(1)(1998) 1-32.

[22] A. Matsumura, T. Nishida, The initial value problem for the equations of motion of viscous and heat-conductive gases, J. Math. Kyoto Univ. 20(1) (1980) 67-104.

[23] A. Matsumura, K. Nishihara, Asymptotics toward the rarefaction wave of the solutions of a one-dimensional model system for compressible viscous gas, Japan J. Appl. Math., 3 (1986), 1-13.

[24] A. Matsumura, K. Nishihara, Global stability of the rarefaction wave of a one-dimensional model system for compressible viscous gas. Comm. Math. Phys. 144(2), (1992), 325-335. 
[25] A. Mellet and A. Vasseur, On the barotropic compressible Navier-Stokes equation, Comm. Partial Differential Equations, 32(3) (2007) 431-452.

[26] A. Mellet and A. Vasseur, Existence and uniqueness of global strong solutions for onedimensional compressible Navier-Stokes equations, SIAM J. Math. Anal. 39 (2008), No. 4, 1344-1365.

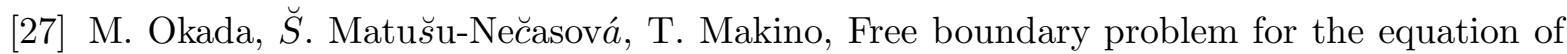
one-dimensional motion of compressible gas with density-dependent viscosity, Ann. Univ. Ferrara Sez. VII (N.S.) 48(2002), 1-20.

[28] I. Straskraba, A. Zlotnik, Global properties of solutions to 1D viscous compressible barotropic fluid equations with density dependent viscosity, Z. Angew. Math. Phys. 54 (2003), no. 4, 593-607.

[29] S. W. Vong, T. Yang, C. J. Zhu, Compressible Navier-Stokes equations with degenerate viscosity coefficient and vacuum II, J. Differential Equations192 (2)(2003) 475-501.

[30] Z. P. Xin, Blow-up of smooth solution to the compressible Navier-Stokes equations with compact density, Comm. Pure Appl. Math. 51(1998) 229-240.

[31] T. Yang, Z. A. Yao, C. J. Zhu, Compressible Navier-Stokes equations with densitydependent viscosity and vacuum , Comm. Partial Differential Equations26 (5-6)(2001) 965-981.

[32] T. Yang, C. J. Zhu, Compressible Navier-Stokes equations with degenerate viscosity coefficient and vacuum, Comm. Math. Phys.230 (2)(2002) 329-363. 